\title{
Mineral cloud and hydrocarbon haze particles in the atmosphere of the hot Jupiter JWST target WASP-43b
}

\author{
Ch. Helling ${ }^{1,2,3}$, Y. Kawashima ${ }^{3}$, V. Graham ${ }^{4}$, D. Samra ${ }^{1,2}$, K. L. Chubb ${ }^{3}$, M. Min $^{3}$, \\ L. B. F. M. Waters ${ }^{3}$, and V. Parmentier ${ }^{5}$ \\ ${ }^{1}$ Centre for Exoplanet Science, University of St Andrews, North Haugh, St Andrews KY16 9SS, UK \\ 2 SUPA, School of Physics \& Astronomy, University of St Andrews, North Haugh, St Andrews KY16 9SS, UK \\ ${ }^{3}$ SRON Netherlands Institute for Space Research, Sorbonnelaan 2, 3584 CA Utrecht, The Netherlands \\ ${ }^{4}$ School of Physics and Astronomy, University of Glasgow, University Avenue, Glasgow G12 8QQ, UK \\ 5 Department of Physics, University of Oxford, Parks Rd, Oxford OX1 3PU, UK
}

e-mail: Christiane.Helling@st-and.ac.uk

Received 31 January 2020 / Accepted 21 May 2020

\begin{abstract}
Context. Having a short orbital period and being tidally locked makes WASP-43b an ideal candidate for the James Webb Space Telescope (JWST) phase curve measurements. Phase curve observations of an entire orbit will enable the mapping of the atmospheric structure across the planet, with different wavelengths of observation allowing different atmospheric depths to be seen.

Aims. We provide insight into the details of the clouds that may form on WASP-43b and their impact on the remaining gas phase, in order to prepare the forthcoming interpretation of the JWST and follow-up data.

Methods. We follow a hierarchical modelling strategy. We utilise 3D GCM results as input for a kinetic, non-equilibrium model for mineral cloud particles and for a kinetic model to study a photochemically-driven hydrocarbon haze component.

Results. Mineral condensation seeds form throughout the atmosphere of WASP-43b. This is in stark contrast to the ultra-hot Jupiters, such as WASP-18b and HAT-P-7b. The dayside is not cloud free but it is loaded with few yet large mineral cloud particles in addition to hydrocarbon haze particles of a comparable abundance. Photochemically driven hydrocarbon haze appears on the dayside, but it does not contribute to the cloud formation on the nightside. The geometrical cloud extension differs across the globe due to the changing thermodynamic conditions. Day and night differ by $6000 \mathrm{~km}$ in pressure scale height. As reported for other planets, the $\mathrm{C} / \mathrm{O}$ is not constant throughout the atmosphere and varies between 0.74 and 0.3 . The mean molecular weight is approximately constant in a $\mathrm{H}_{2}$ dominated WASP-43b atmosphere because of the moderate day/night-temperature differences compared to the super-hot Jupiters. Conclusions. WASP-43b is expected to be fully covered in clouds which are not homogeneously distributed throughout the atmosphere. The dayside and the terminator clouds are a combination of mineral particles of locally varying size and composition as well as of hydrocarbon hazes. The optical depth of hydrocarbon hazes is considerably lower than that of mineral cloud particles such that a wavelength-dependent radius measurement of WASP-43b would be determined by the mineral cloud particles but not by hazes.
\end{abstract}

Key words. planets and satellites: atmospheres - planets and satellites: gaseous planets - astrochemistry - solid state: refractory planets and satellites: individual: WASP-43b

\section{Introduction}

WASP-43b is a giant gas planet that has been selected as a James Webb Space Telescope (JWST) target because it orbits a near-solar abundance $\mathrm{K} 7 \mathrm{~V}$ star $\left(T_{\text {eff }}=4500 \mathrm{~K}\right)$ at an orbital distance of $a \approx 0.0153$ au in 0.813 days (Gillon et al. 2012). Its atmosphere can therefore be well characterised through transmission spectroscopy and thermal emission measurements. The dayside of WASP-43b is suggested to have a black-body equivalent temperature of $\approx 1700 \pm 200 \mathrm{~K}$ and an equilibrium temperature of $T_{\mathrm{eq}}=1400 \mathrm{~K}$ (Blecic et al. 2014). With a planetary mass of $1.78 \pm 0.01 M_{\mathrm{J}}$ and a radius of $R_{\mathrm{P}}=0.93 \pm 0.09 R_{\mathrm{Jup}}$ (Hellier et al. 2011), its bulk density is $2.744 \pm 0.4 \mathrm{~g} \mathrm{~cm}^{-3}$. Applying retrieval approaches, $\mathrm{H}_{2} \mathrm{O}$ (Kreidberg et al. 2014; Stevenson et al. 2017) and CO (Feng et al. 2016) are suggested to be present in the atmospheric gas that is accessible from thermal emission (but no $\mathrm{CH}_{4}$ as of yet). A comprehensive summary of observational results for WASP-43b can be found in Stevenson et al. (2017) who present a full-orbit phase curve in the warm Spitzer bands 3.6 and $4.5 \mu \mathrm{m}$. WASP-43b is classified as a giant gas planet similar to HD 189733b and HD 209458b but it appears to show less day-night heat distribution (Komacek et al. 2017) despite orbiting closer to its host star compared to HD 189733b $(a \approx 0.0031 \mathrm{au})$ and HD 209458b $(a \approx 0.0047 \mathrm{au})$. We note that both, HD 189733b and HD 209458b, have smaller mean bulk densities of 1.8 and $0.616 \mathrm{~g} \mathrm{~cm}^{-3}$, respectively (Table 1 in Helling et al. 2016). Kataria et al. (2015) and Stevenson et al. (2014, 2017) point out that the day-side phase curve can be fitted with a cloudfree Global Circulation Model (GCM), but such a GCM predicts too high of a flux on the nightside. Mendonça et al. (2018a) discuss non-equilibrium chemistry caused by advection for $\mathrm{CO}$, $\mathrm{CO}_{2}, \mathrm{H}_{2} \mathrm{O}$ and $\mathrm{CH}_{4}$, suggesting a general advection driven overabundance of $\mathrm{CO}$ and $\mathrm{H}_{2} \mathrm{O}$ for a high $\mathrm{C} / \mathrm{O}=2.0$ and for $\mathrm{CH}_{4}$ on the night side if $\mathrm{C} / \mathrm{O}=0.5$. Morello et al. (2019) reanalysed the WASP-43b data, suggesting a nightside brightness between the results of Stevenson et al. (2017) and Mendonça et al. (2018a). Chubb et al. (2020) performed a re-analysis of the HST/WFC3 and Spitzer/IRAC transmission data for WASP-43b using all available opacity data, and they report the detection of $\mathrm{AlO}$ and $\mathrm{H}_{2} \mathrm{O}$ in transmission with a high statistical significance. No 
evidence for the presence of $\mathrm{CO}, \mathrm{CO}_{2}$, nor $\mathrm{CH}_{4}$ in transmission could be reported; however, the study notes the small wavelength region analysed. The presence of $\mathrm{AlO}$ points to a rich atmosphere chemistry in WASP-43b, possibly leading to complex metal-oxide clusters similar to asymptotic giant branch (AGB) star envelopes (Decin et al. 2017). Venot et al. (2020) present the JWST WASP-43b atmosphere consortium paper with predictions for atmospheric structures, the most important kinetic gas-phase species, and cloud properties.

In this paper, we apply a hierarchical modelling approach where we study kinetic cloud formation, kinetic gas and hydrocarbon haze chemistry by utilizing the output from a 3D GCM for WASP-43b. We demonstrate that WASP-43b is not cloud free. We model the formation and explore the properties of two chemically different cloud particle ensembles, mineral cloud particles and hydrocarbon hazes, that form by distinctly different processes. One of the most remarkable differences is that mineral cloud particles form from an oxygen-rich gas and that hydrocarbon hazes require the presence of the hydrocarbon precursors such as $\mathrm{CH}_{4}$ in the same oxygen-rich, atmospheric environment. We make suggestions about their composition, particle sizes and derive implications for the gas phase composition to be expected in chemical equilibrium, but also for the $\mathrm{C} / \mathrm{H} / \mathrm{O} / \mathrm{N}$ chemistry calculated from photochemical modelling.

Our results suggest that WASP-43b is covered in clouds, a finding similar to HD 189733b and HD 209458b, and hazes that mainly occur on the dayside and in the terminator regions. We suggest that spectra and phase-curves of irradiated exoplanets, and giant gas planet like WASP-43b in particular, may be confused by the co-existence of hydrocarbon haze particles and mineral seed particles in the upper most atmosphere that is accessible by transmission spectroscopy. These are atmospheric regions of low gas density where photochemical processes enable the formation of hydrocarbon haze particles but where surface growth processes are still far too inefficient to cause a substantial growth of the mineral particles (in fact, of any particle).

Section 2 summarises our approach and provides necessary details to allow a comparison to our previous modelling method. Section 3 gives an account of the temperature structure which is a major driver of the cloud formation. Section 4 presents our analysis of the kinetically forming mineral clouds in WASP-43b. Section 5 summarises our general findings for the gas-phase composition assuming chemical equilibrium. Here, we discuss the validity of assuming a constant mean molecular weight, the carbon-to-oxygen ratio and the degree of ionisation. Section 6 addresses the effect of mixing processes on the abundance of $\mathrm{C} / \mathrm{H} / \mathrm{O} / \mathrm{N}$-binding molecules and the formation of hydrocarbon hazes under the effect of the host star's radiation. Section 7 presents the comparison between the models applied here leading to mineral particles and hydrocarbon haze and Appendix A addresses the challenge of systematic errors within cloud property comparisons from different model approaches. Section 8 concludes the paper.

\section{Approach}

We adopt a hierarchical three-model approach in order to examine the cloud structure on WASP-43b similar to works on the hot Jupiters HD 189733b and HD 209458b (Lee et al. 2015; Helling et al. 2016) and the ultra-hot Jupiters WASP18b (Helling et al. 2019a) and HAT-P-7b (Helling et al. 2019b; Molaverdikhani et al. 2020): The first modelling step produces pre-calculated, cloud-free 3D GCM results. These results are
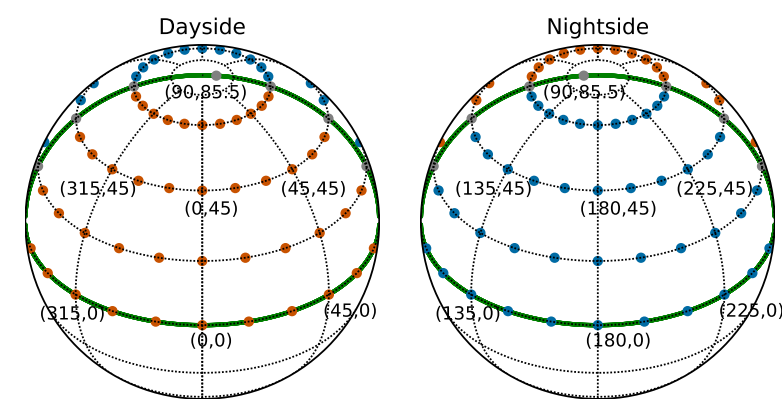

Fig. 1. Positions of the 1D profiles taken from the GCM (left: dayside; right: nightside), with the points $(\phi, \theta)=\left(0^{\circ}, 0^{\circ}\right)$ and $(\phi, \theta)=\left(180^{\circ}, 0^{\circ}\right)$ being the sub-stellar (phase $=0.5$ ) and anti-stellar points, respectively $((\phi, \theta)=($ longitudes, latitudes $))$. 1D profiles that sample the dayside (red), nightside (blue), morning and evening terminator (both grey) are indicated. The green lines show the locations of slices used in subsequent maps along the equator $\left(\theta=0^{\circ}\right)$ and the terminator (longitudes $\phi=90^{\circ}-$ evening terminator, $\phi=270^{\circ}-$ morning terminator).

used as input for the second modelling step which is a kinetic cloud formation model consistently combined with equilibrium gas-chemistry calculations. We utilise $971 \mathrm{D}\left(T_{\mathrm{gas}}(z), p_{\mathrm{gas}}(z)\right.$, $\left.v_{z}(x, y, z)\right)$-profiles for WASP-43b similar to our work on HAT-P$7 \mathrm{~b}$ as visualised in Fig. 1: $T_{\text {gas }}(z)$ is the local gas temperature [K], $p_{\text {gas }}(z)$ is the local gas pressure [bar] and $v_{z}(x, y, z)$ is the local vertical velocity component $\left[\mathrm{cm} \mathrm{s}^{-1}\right]$. The same $1 \mathrm{D}$ profiles are used as input for our third modelling step, the kinetic haze simulation (Kawashima \& Ikoma 2018, 2019; Kawashima et al. 2019). The same, height-dependent mixing coefficient $K_{\mathrm{zz}}$, which is assumed as $H v_{\mathrm{z}}$ with $H$ being the atmospheric scale height, is used in both the kinetic cloud formation and the kinetic haze formation model. We use the solar element abundances from Asplund et al. (2009) for the undepleted element abundances.

The hierarchical approach has the limitation of not explicitly taking into account the potential effect of horizontal winds on cloud formation. However, processes governing the formation of mineral clouds are determined by local thermodynamic properties which are the result of 3D dynamic atmosphere simulations. Horizontal transport can affect both the cloud formation and the cloud properties. Figure 4 in Lee et al. (2015) shows for the gas giant HD 189733b (orbiting a similar host star to that of WASP-43b but at a larger orbital distance of 0.0314 AU) that the (vertical) settling of the cloud particles occurs at time scales longer than a typical (horizontal) advection time scale on the nightside but that the settling and advection are of comparable magnitudes on the dayside. Cloud particle properties such as particle size or particle composition should be smeared out in longitude compared to the results shown here. We note that comparing Lee et al. (2015) (without horizontal advection) and Lee et al. (2016) (including horizontal advection), the noncoupled problem is both more computationally feasible, easier to interpret and provides reasonable first order insights into the expected mean cloud particle size and composition, cloud distribution and gas phase depletion in exoplanet atmospheres. The situation is somewhat different for photochemically triggered cloud formation. Photochemical hydrocarbon-haze production, for example, is determined by the external radiation field. A certain smearing out of the hydrocarbon haze properties should also be expected for this cloud particle population in particular in transient regimes like at the day-night terminators. Agúndez et al. (2014) and Drummond et al. (2018) demonstrate from HD 189733b and HD 209458b simulations that longitudinal quenching of $\mathrm{C} / \mathrm{H} / \mathrm{O} / \mathrm{N}$ species occurs inside the jet regions and 
on the nightside. Molaverdikhani et al. (2020) point out for HAT-P-7b, however, that the atmospheric optical depth is little effected, in particular if cloud particles form through other paths than $\mathrm{C} / \mathrm{H} / \mathrm{O} / \mathrm{N}$ chemistry.

3D GCM input. We use the SPARC/MITgem (Showman et al. 2009) to determine the 3D thermal structure of WASP43b. The model solves the primitive equations coupled to non-grey radiative transfer. We assume solar composition abundances including rainout of condensates but we do not consider any cloud radiative feedback. Local chemical equilibrium is assumed, since, as shown by Steinrueck et al. (2019), the effect of disequilibrium chemistry on the thermal structure is small compared to the horizontal temperature contrasts. More details of the specific model run can be found in Parmentier et al. (2018) and Irwin et al. (2020). For more details on the GCM approach, we refer the reader to the description in Helling et al. (2019b). The outputs of our WASP-43b models are very similar to the ones of Kataria et al. (2015) since both are utilising the same base 3D GCM code.

We ran the simulation for 300 Earth days and averaged all physical quantities over the last 100 days of the simulation. The deeper atmosphere would need a much longer, impractical integration time to converge. Our results therefore rely on the assumption that the deep atmospheric properties do not significantly alter the photospheric layers, an often made and reasonable assumption as shown by Amundsen et al. (2016). Although this has recently been called into question in the context of grey radiative transfer schemes (Carone et al. 2020; Wang \& Wordsworth 2020), the timescales needed for equilibration (50 000-100 000 days) are impractical when using non-grey radiative transfer.

Venot et al. (2020) show in their Fig. 9 that the cloudless GCM spectra fit very well to the dayside atmosphere but is a poor fit to the nightside atmosphere. In Fig. 11, they show that post-processed clouds allow a much better fit to the nightside atmospheres. When active clouds are added in the GCM the nightside spectra does not change significantly compared to the post-processed case, showing that the nightside temperature structure is not extremely affected. This experiment shows that the cloudless GCM is a reasonable approximation to the temperature map of WASP-43b.

Kinetic cloud formation. To preserve consistency, we apply the same set-up of our kinetic cloud formation model (nucleation, growth, evaporation, gravitational settling, element consumption and replenishment) and equilibrium gas-phase calculations like for HAT-P-7b (Sect 2.1 in Helling et al. 2019b). In addition, we include $\mathrm{KCl}$ as nucleation and growth species. The seed forming species need to be considered as surface growth material, too, since both processes (nucleation and growth) compete for the participating elements ( $\mathrm{Ti}, \mathrm{Si}, \mathrm{O}, \mathrm{C}$, $\mathrm{K}$ and $\mathrm{Cl}$ in this work). We consider the formation of 16 bulk materials ( $\mathrm{s}=\mathrm{TiO}_{2}[\mathrm{~s}], \mathrm{Mg}_{2} \mathrm{SiO}_{4}[\mathrm{~s}], \mathrm{MgSiO}_{3}[\mathrm{~s}], \mathrm{MgO}[\mathrm{s}], \mathrm{SiO}[\mathrm{s}]$, $\mathrm{SiO}_{2}$ [s], $\mathrm{Fe}[\mathrm{s}], \mathrm{FeO}[\mathrm{s}], \mathrm{FeS}[\mathrm{s}], \mathrm{Fe}_{2} \mathrm{O}_{3}[\mathrm{~s}], \mathrm{Fe}_{2} \mathrm{SiO}_{4}$ [s], $\mathrm{Al}_{2} \mathrm{O}_{3}$ [s], $\mathrm{CaTiO}_{3}[\mathrm{~s}], \mathrm{CaSiO}_{3}[\mathrm{~s}], \mathrm{C}[\mathrm{s}], \mathrm{KCl}[\mathrm{s}]$; s indicating that this is a condensate material) that form from 11 elements $(\mathrm{Mg}, \mathrm{Si}, \mathrm{Ti}$, $\mathrm{O}, \mathrm{Fe}, \mathrm{Al}, \mathrm{Ca}, \mathrm{S}, \mathrm{C}, \mathrm{K}$ and $\mathrm{Cl}$ ) by 128 surface reactions. The abundance of these 11 elements will decrease if cloud particles are forming (nucleation, growth) and increase if cloud particles evaporate. Sulphur has not been included in our present mineral cloud model. Sulphuric materials in form of S[s], FeS[s], MgS[s] would contribute by less than $10 \%$ in volume fraction in a solar element abundance gas (see Fig. 6 in Helling 2019).
Kinetic haze formation. We perform the simulations of photochemistry and haze formation using the models of Kawashima \& Ikoma (2018). The procedure is the same as our previous studies (Kawashima et al. 2019; Kawashima \& Ikoma 2019): we first perform photochemical simulations to derive the steady-state distribution of gaseous species. Then, assuming the production rate of haze monomers at each altitude as the sum of the photodissociation rates of the major hydrocarbons in our photochemical model, $\mathrm{CH}_{4}, \mathrm{HCN}$ and $\mathrm{C}_{2} \mathrm{H}_{2}$, we derive the steady-state distribution of haze particles by the particle growth simulations. In the particle growth simulation, distribution of haze particles is derived considering coagulation of homo-disperse monomers, gravitational sedimentation and diffusion. Neither evaporation nor condensation is considered. Thermal decomposition such as considered in Lavvas \& Koskinen (2017) can be important in such a hot atmosphere of WASP-43b, but it is ignored due to the uncertainty of the thermal stability of haze. We adopt the haze monomer radius of $1 \mathrm{~nm}$ and its density of $1 \mathrm{~g} \mathrm{~cm}^{-3}$. The haze formation feedback on the atmospheric chemistry such as the elemental depletion is not considered due to the high computational cost. For the details of the photochemical and particle growth models, see Kawashima \& Ikoma (2018).

We choose four representative points along the equator $\left(\theta=0^{\circ}\right)$ to perform the haze formation simulations because of the high computational cost; sub-stellar point $\left(\phi=0^{\circ}\right)$, evening terminator $\left(\phi=90^{\circ}\right)$, anti-stellar point $\left(\phi=180^{\circ}\right)$ and morning terminator $\left(\phi=270^{\circ}\right)$. For the anti-stellar point $\left(\phi=180^{\circ}\right)$, we only perform the chemical simulation and do not perform the haze formation calculation because of the absence of a sufficient radiation field and hence, no photochemistry.

As for the elemental abundance ratios used in the photochemical calculation, we adopt the original solar ones. Though we have also simulated the photochemistry using the reduced oxygen abundance as a result of mineral cloud formation, we have confirmed that the depletion of the oxygen abundance in the cloud-forming region is replenished by the vertical eddy diffusion transport from the lower atmosphere with the solar abundance. However, if we are able to couple mineral cloud formation with photochemical simulation, the oxygen abundance in the cloud-forming region should be kept depleted because of the much shorter timescale of cloud formation compared to that of diffusion. Coupling those two simulations is our future work. For the UV spectrum of WASP-43, since it has not been observed, we use that of HD 85512 constructed by the MUSCLES Treasury Survey (France et al. 2016; Youngblood et al. 2016; Loyd et al. 2016) because of its similar stellar type to WASP-43. The attenuation of the UV spectrum due to the wavelength-dependent molecular absorption at each altitude is taken into account. For the simulation of the sub-stellar point, we ignore the diurnal variation factor of $1 / 2$ in the expression of the photodissociation rate (see Kawashima \& Ikoma 2018, Eq. (3)), which we adopt for the terminator points and for that of the anti-stellar point, we ignore photochemistry. Finally, as for the zenith angle of the stellar irradiation, which should be $0.0^{\circ}$ for the sub-stellar point while the value somewhat smaller than $90.0^{\circ}$ for the terminator if the host star is spatially resolved, we use the globally-averaged value of $57.3^{\circ}$ (Hu et al. 2012) for all the points for simplicity.

\section{The warm atmosphere of WASP-43b}

Figure 2 (left) visualises the thermodynamic input that we utilise for our following study of cloud and haze formation and the gasphase composition in the atmosphere of WASP-43b. WASP-43b 


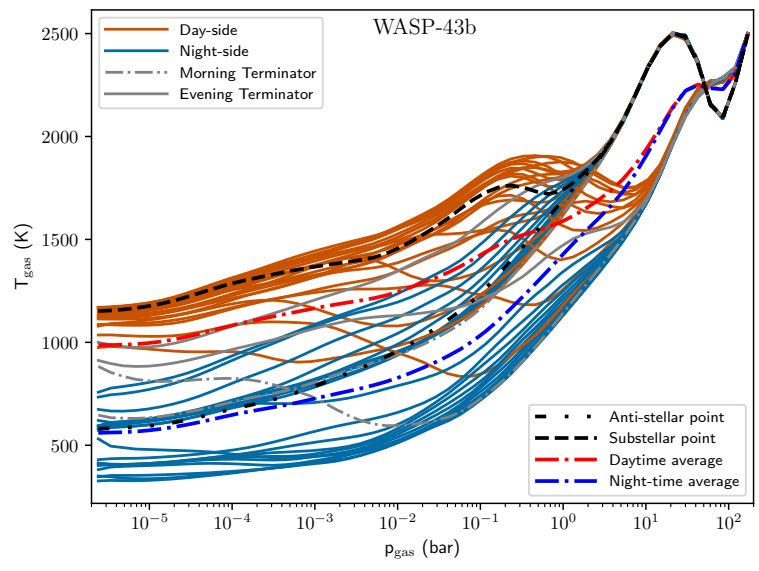

Equatorial Slice $\left(\theta=0.0^{\circ}\right)$

90.0
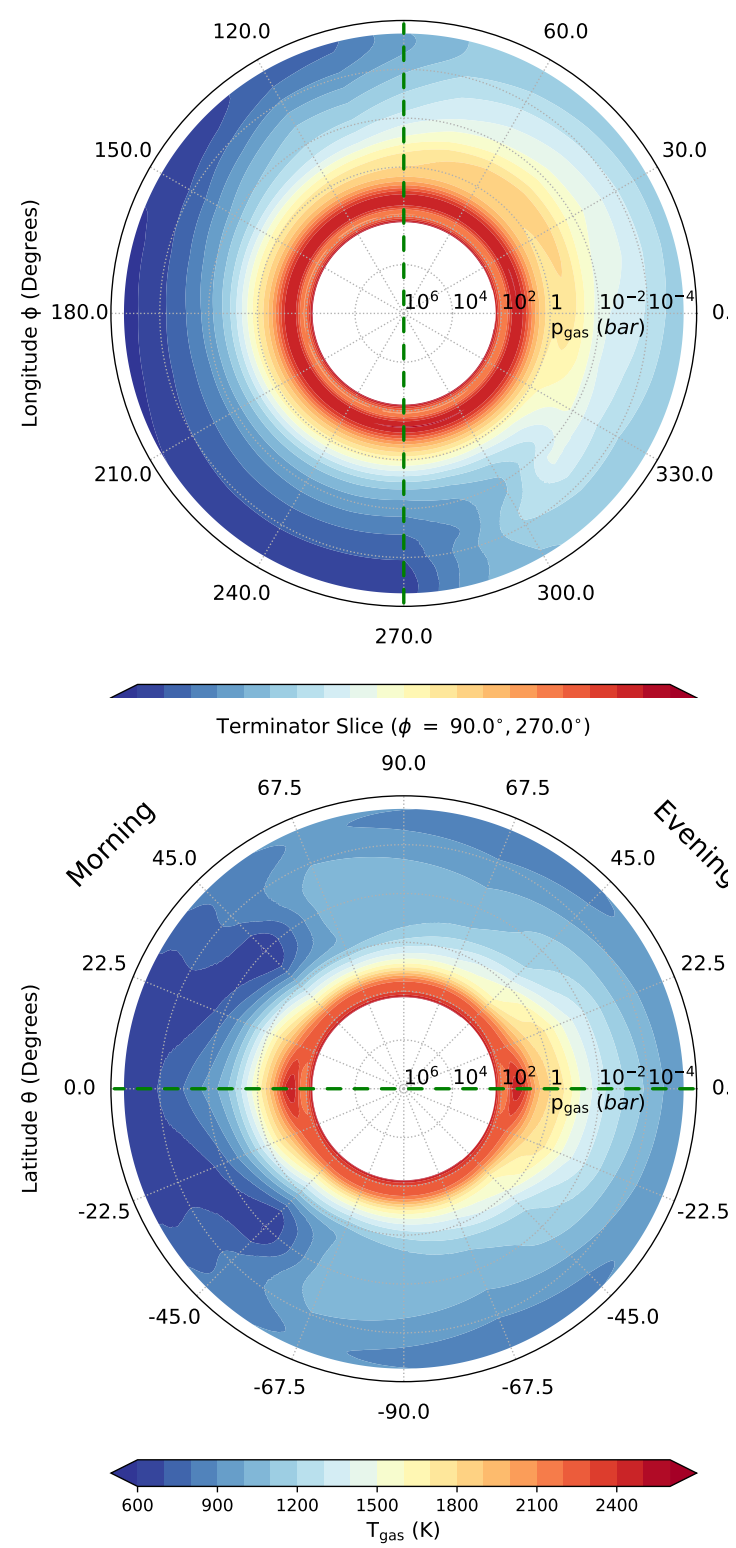

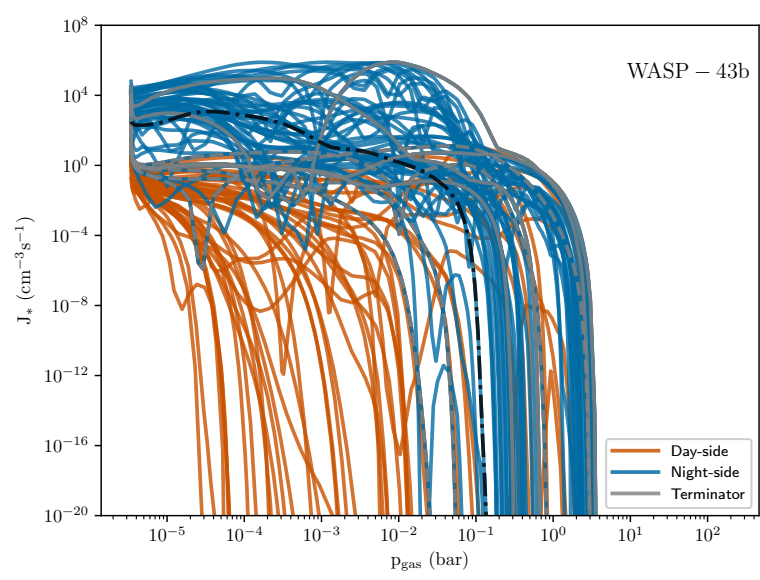

Equatorial Slice $\left(\theta=0.0^{\circ}\right)$
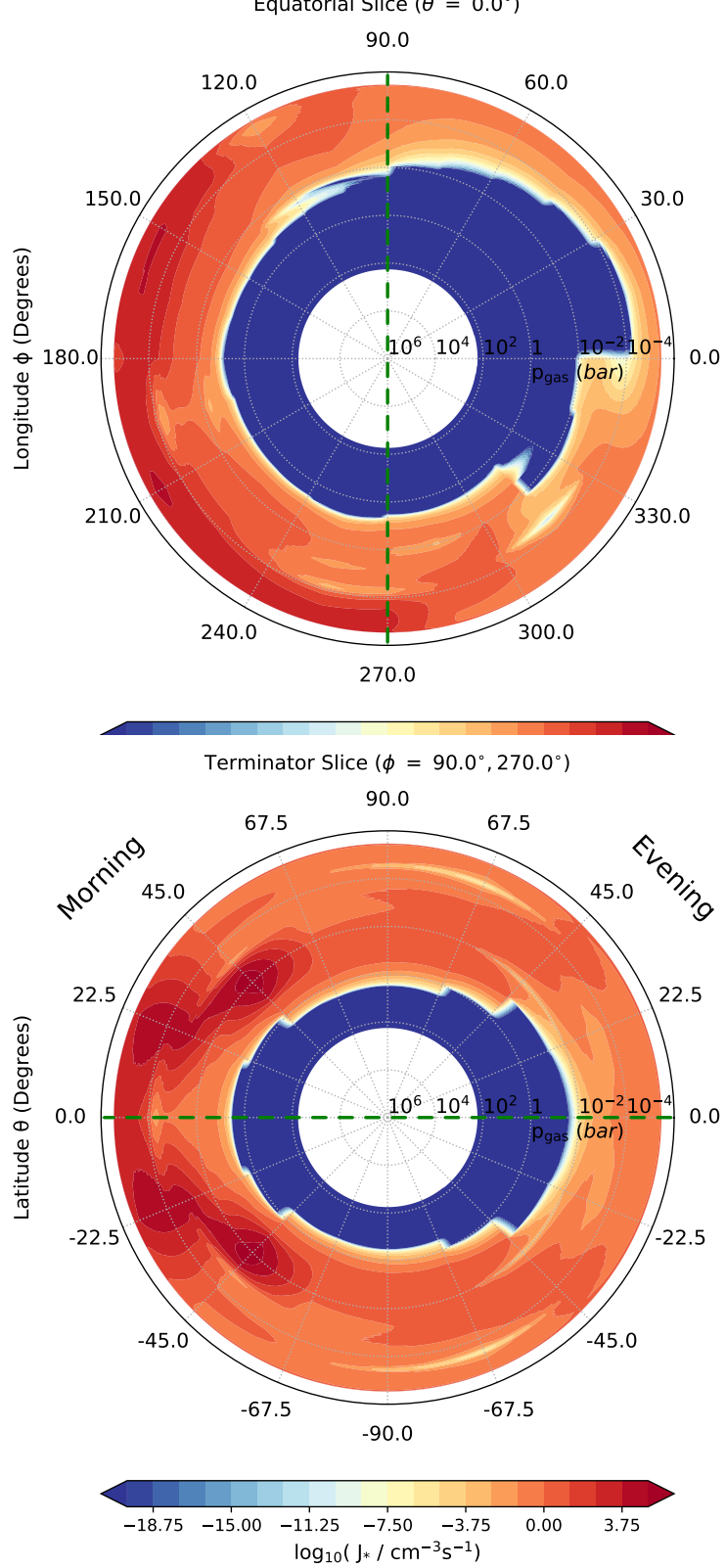

Fig. 2. Left: local gas temperature and local gas pressure $\left(T_{\text {gas }}, p_{\text {gas }}\right)$ extracted from the 3D GCM (Parmentier et al. 2018). Right: total mineral seed formation (nucleation) rate, $J_{*}\left[\mathrm{~cm}^{-3} \mathrm{~s}^{-1}\right]$. Top: 1D profiles for the probed 97 profiles (red - dayside, blue - nightside, grey dashed morning terminator $\left(\phi=270^{\circ}\right)$, grey-solid - evening terminator $\left(\phi=90^{\circ}\right)$, black dashed - substellar point $\left(\phi=0^{\circ}\right)$ black dot-dashed - antistellar point $\left(\phi=180^{\circ}\right)$ ). Middle: $2 \mathrm{D}$ cut visualising the temperature distribution (left) and the distribution of the formed condensation seeds $(r i g h t)$ in the equatorial plane. Bottom: $2 \mathrm{D}$ cut along the terminator for the a tidally locked WASP-43b. 


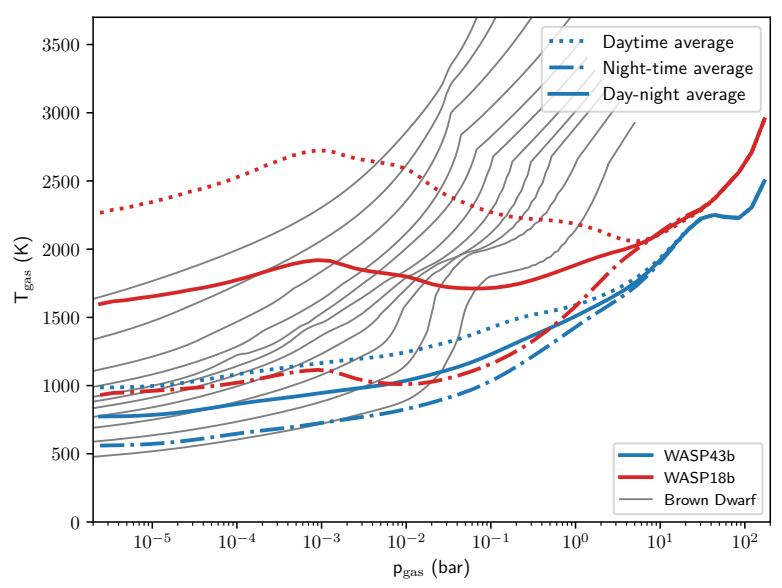

Fig. 3. Comparison of WASP-43b to the ultra-hot Jupiter WASP-18b (red) and DRIFT-PHOENIX young planets/brown dwarf atmosphere structures for $\log (g)=3.0$ and $T_{\text {eff }}=1000-3000 \mathrm{~K}$ by steps of $200 \mathrm{~K}$ (Witte et al. 2009).

is a hot Jupiter that is assumed to be tidally locked with its host star which results in a dayside that is hotter than the nightside. The resulting gas temperature difference of $\approx 700 \mathrm{~K}$ at a gas pressure of $\approx 10^{-4}$ bar drives a global circulation from the day to the nightside (Parmentier et al. 2018; Mendonça et al. $2018 \mathrm{~b}$ ). A smaller temperature difference is suggested between the substellar and anti-stellar point (top left) and the terminator profiles. We also show the dayside (red dash-dot) and the nightside (blue dash-dot) averages which are poor representations of the day- and the nightside temperature distributions, respectively. The inspection of the equatorial temperature distribution (Fig. 2 bottom left) suggests a west-east asymmetry with the east $\left(\phi \rightarrow 90^{\circ}\right)$ being warmer than the westerly $\left(\phi \rightarrow 270^{\circ}\right)$ dayside. The day-night temperature difference can clearly be seen from this $2 \mathrm{D}$ plots and the changing temperature throughout the whole terminator regions that is probed by transmission spectroscopy is best seen in Fig. 2 (middle left).

Setting the JWST target WASP-43b in perspective, we further demonstrate that, within the uncertainty of the GCM temperature structures, WASP-43b's day- and nightside are on average cooler than those of an ultra-hot Jupiter-like WASP-18b (Fig. 3). We also note that the vertical extension of the GCM profiles fall short of what 1D atmosphere simulations can provide by comparing to our 1D DRIFT-PHOENIX model (Fig. 3, solid grey lines). Carone et al. (2020) have demonstrated the effect of the inner boundary on the flow regimes in the atmosphere of WASP-43b. This has substantial impact also on the local thermodynamic conditions inside the atmosphere and we will need to return to this challenge in due time.

\section{A theoretical analysis of kinetically forming, mineral clouds on WASP-43b}

In this section, we discuss the principle results for mineral cloud formation in the hot Jupiter WASP-43b to enable a detailed insight into the cloud properties that will shape the JWST spectrum of WASP-43b. In Sect. 6, we show in how far photochemical hydrocarbon haze formation does complete this picture of cloud formation in the atmosphere of WASP-43b leading to the idea of the possible presence of two chemically distinct cloud ensembles if photochemical processes affect the chemical carbon reservoir of the atmosphere (Sect. 7).
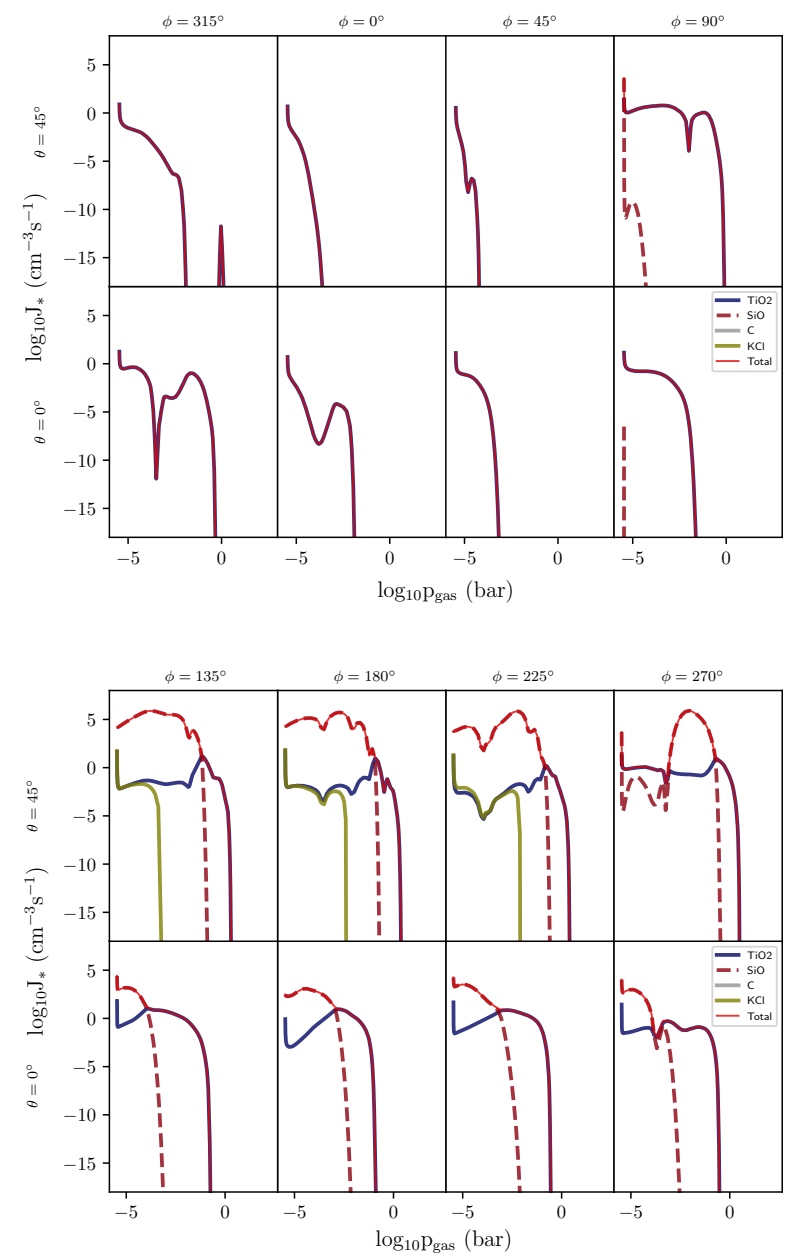

Fig. 4. Total seed formation (nucleation) rate, $J_{*}=\Sigma J_{\mathrm{s}}\left[\mathrm{cm}^{-3} \mathrm{~s}^{-1}\right]$ and the individual nucleation rates, $J_{\mathrm{s}}\left[\mathrm{cm}^{-3} \mathrm{~s}^{-1}\right]$, for $\mathrm{s}=\mathrm{TiO}_{2}, \mathrm{SiO}, \mathrm{KCl}$, $\mathrm{C}$ on four selected (longitude, latitude) 1D profiles from the 3D GCM (Parmentier et al. 2018) for WASP-43b. Top: dayside. Bottom: nightside.

Firstly, we observe that nucleation seeds form for all the 1D profiles used here to probe cloud formation in the 3D atmosphere of WASP-43b (Fig. 2, right, top). This is in stark contrast to the ultra-hot Jupiters, like WASP-18b and HAT-P7b, but in line with other hot Jupiters like HD 189733 b and HD 209458 b. Secondly, the rate of seed formation differs strongly between the day and the nightside because of the different thermodynamic conditions. The seed formation is more efficient on the nightside where $\mathrm{SiO}$ seeds dominate in the low-pressure part of the atmosphere. Once $\mathrm{SiO}$ [s] surface growth kicks in efficiently, $\mathrm{SiO}$ nucleation is terminated and $\mathrm{TiO}_{2}$ seeds form more efficiently in the middle atmosphere at $\approx 1$ bar. $\mathrm{KCl}$ seeds do form on the night side in the low-pressure atmosphere but with a rate that is too low to be significant (Fig. 4)

\subsection{Asymmetric cloud coverage and non-homogeneous cloud properties}

The equatorial cut (Fig. 2, middle right) shows the day-night and west-east $\left(\phi=270^{\circ} \rightarrow 90^{\circ}\right)$ asymmetry in the formation rate of condensation seeds in the atmosphere of WASP-43b. The equatorial asymmetry in the seed formation rate is less strong (Fig. 2, bottom). Based on the present 1D profiles, the eastern dayside of WASP-43b $\left(\phi=0^{\circ} \rightarrow 90^{\circ}\right)$ forms condensation seeds in a narrow range at $p_{\text {gas }}<10^{-4}$ bar. The western dayside $\left(\phi=0^{\circ} \rightarrow 270^{\circ}\right)$ 


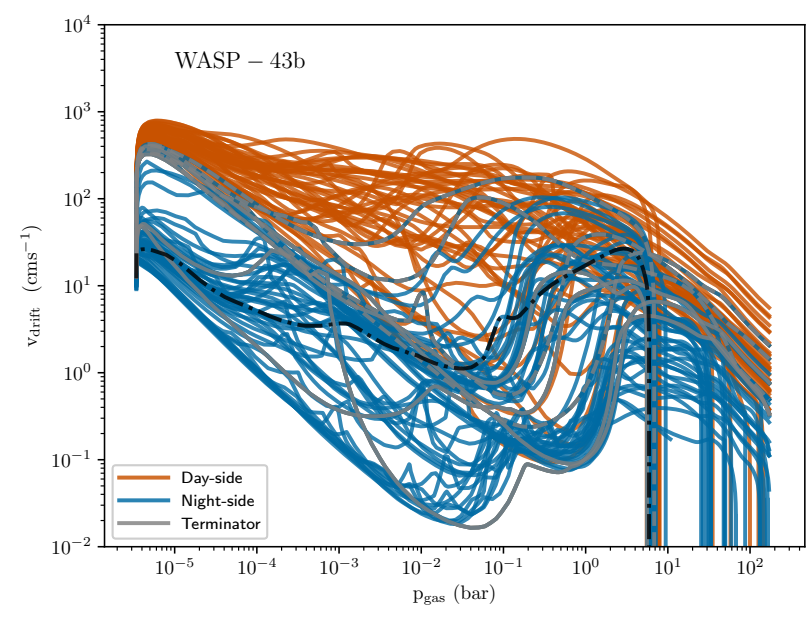

Fig. 5. Drift velocity for all 1D trajectories of WASP-43b. The nightside profiles are shown in blue, the dayside profiles in orange, the terminator profiles in grey.

features a wider seed formation zone compared to the eastern dayside which deepens with increasing longitude $\phi$ in the equatorial region of WASP-43b.

The lower boundary of the seed formation region (see Fig. 2, right) does not coincide with the lower boundary of the cloud (compare Fig. 6, left) because the particles grow and fall (gravitational settle) through the atmosphere into deeper, warmer atmospheric regions of increasing pressure. The growth in these warm regions is supported by the increasing gas pressure which increases the collision rates. The higher pressures further stabilise materials that already formed at high temperatures. The inner cloud boundary is set by the thermal stability of the particles of a particular size; big particles survive longer as they have more material to evaporate.

Figure 6 (left) demonstrates that the cloud particles sizes are not homogeneous but they change for all 1D profiles probed in our study. Figure 6 further demonstrates that cloud particles are present thought almost the entire atmospheric volume sampled here, in particular also on both the day- and the nightside. The characteristic cloud properties, however, are different between the day- and the nightside. As consequence of the seed formation discussed with regard to Fig. 2 (right), the mean cloud particle sizes are larger over a larger pressure range on the easterly dayside $\left(\phi=0^{\circ} \rightarrow 90^{\circ}\right)$ compared to the westerly dayside $\left(\phi=0^{\circ} \rightarrow 270^{\circ}\right)$ and the nightside (Fig. 6, middle left). A similar but less pronounced asymmetry occurs for the terminator regions (Fig. 6, bottom left).

The cloud opacity is determined by the cloud particles sizes, their abundance and their material properties. The dustto-gas ratio, $\rho_{\mathrm{d}} / \rho_{\text {gas }}$ (Fig. 6 , right) combines these properties and visualises where the highest cloud mass load occurs in an atmosphere. $\rho_{\mathrm{d}} / \rho_{\mathrm{gas}}$ shows that only the inner part of the WASP$43 \mathrm{~b}$ atmosphere is cloud-free. The largest $\rho_{\mathrm{d}} / \rho_{\text {gas }}$ occurs on the nightside and reaches well across the morning terminator at $\phi=270^{\circ}$. The dayside is not cloud free but has a considerably lower cloud load, hence, it might be mistakenly interpreted as cloud-free.

Simple time scale estimate shows that cloud particle of sizes $\langle a\rangle \approx 0.001-0.1 \mu \mathrm{m}$ (Fig. 6) occurring in the terminator and dayside regions remain in the atmospheric regions accessible to transmission spectroscopy of $p_{\text {gas }} \approx 10^{-5}-10^{-4}$ bar for about $2 \mathrm{~h}$, using $H \approx 8 \times 10^{6} \mathrm{~cm}$ (Fig. 9) and $v_{\mathrm{dr}}=10^{3} \mathrm{~cm} \mathrm{~s}^{-1}$ (Fig. 5). With a slightly smaller nightside scale height of $H \approx 3 \times 10^{6} \mathrm{~cm}$ and $v_{\mathrm{dr}}=5 \mathrm{~cm} \mathrm{~s}^{-1}$, the respective cloud particles that formed in the low-pressure regions of $p_{\text {gas }} \approx 10^{-5}-10^{-4}$ bar and grew to a mean particle size of only $\langle a\rangle \approx 10^{-3} \mu \mathrm{m}$ remain $\approx 170 \mathrm{~h}$ (or $\approx 7 \mathrm{~d}$ with a day of $24 \mathrm{~h}$ ). Once the nightside particles have sank into the deeper atmosphere, the time scale decreases considerably as their size increases by orders of magnitudes and, although time scale vary throughout the atmosphere, the settling time scale become comparable to the dayside example stated above.

\subsection{General material composition of cloud particles}

Figure 7 demonstrates that the material composition of the cloud particles on WASP-43b does not change dramatically between the day- and the nightside in the low pressure regions where $p_{\text {gas }} \approx 10^{-6}-10^{-4}$ bar for the $1 \mathrm{D}$ profiles inspected in this study: the dominating materials are silicates $\left(\mathrm{MgSiO}_{3}[\mathrm{~s}]\right.$, $\mathrm{Mg}_{2} \mathrm{SiO}_{4}$ [s], $\left.\mathrm{Fe}_{2} \mathrm{SiO}_{4}[\mathrm{~s}], \mathrm{CaSiO}_{3}[\mathrm{~s}]\right)$ and metal oxides (MgO[s], $\mathrm{SiO}[\mathrm{s}], \mathrm{SiO}_{2}[\mathrm{~s}], \mathrm{FeO}[\mathrm{s}], \mathrm{Fe}_{2} \mathrm{O}_{3}$ [s], $\mathrm{CaTiO}_{3}[\mathrm{~s}]$ ). Silicates dominate the cloud particle volume by far with values of $45-60 \%$. High temperature condensates $\left(\mathrm{TiO}_{2}[\mathrm{~s}], \mathrm{Fe}[\mathrm{s}], \mathrm{Al}_{2} \mathrm{O}_{3}[\mathrm{~s}]\right)$ dominate the cloud particle volume at higher temperatures where silicates have evaporated. Carbon contributes a little bit and salts (here $\mathrm{KCl}[\mathrm{s}]$ ) play no significant role for the material composition in the temperature ranges of the WASP-43b atmosphere investigated here. $\mathrm{KCl}$ did occur as a minor nucleation species in the northern hemisphere of the nightside of WASP-43b. A detailed account of the individual contributions of the 16 materials considered in our study is given in Fig. B.2 for the two terminators, the substellar and the anti-stellar point. We note that the terminator structures are rather comparable in the frame of the models applied here.

\subsection{Column integrated cloud properties}

Column integrated cloud properties may enable us to compare complex information without compromising the information content too much. All three characteristic cloud properties, nucleation rate, mean particles size and material composition, can change throughout a planetary atmosphere because the thermodynamic conditions change vertically and horizontally. This has been demonstrated for WASP-43b in the previous sections. The vertical structures have been shown to be very inhomogeneous such that not one value suffices to describe exoplanet clouds and the same holds for the horizontal cloud distributions for which we have not only demonstrated a large day-night difference but our model suggests also a west-east change in cloud properties on the dayside of WASP-43b. Despite this complexity, column integrated mean values of selected cloud properties do recover our major findings from the previous, more detailed discussions as follows.

Figure 8 (top) shows the column integrated total nucleation rate $\int_{z \min }^{\mathrm{zmax}} J_{*}(z) \mathrm{d} z\left(\mathrm{~cm}^{-2} \mathrm{~s}^{-1}\right)$ with $J_{*}=\sum_{\mathrm{s}} J_{\mathrm{s}}\left(s=\mathrm{TiO}_{2}\right.$, $\mathrm{SiO}, \mathrm{C}, \mathrm{KCl}), z_{\min }$ and $z_{\max }$ spanning the geometric extension of the atmosphere at each of 18 (out of 97) selected (longitudes, latitude)-profiles. Figure 8 (middle) provides the height-averaged, number density weighted cloud mean particle size $\langle\langle a\rangle\rangle=\left(\int_{\text {zmin }}^{\text {zmax }} n_{\mathrm{d}}(z)\langle a(z)\rangle \mathrm{d} z\right) /\left(\int_{\mathrm{zmin}}^{\mathrm{zmax}} n_{\mathrm{d}}(z) \mathrm{d} z\right)[\mu \mathrm{m}]$ with $n_{\mathrm{d}}(z)\left[\mathrm{cm}^{-3}\right]$ the local cloud particle number density and $\langle a(z)\rangle$ the mean particle size as defined in Eq. (A.3). Figure 8 very clearly shows the anti-correlation between the nucleation rate (top) and the mean cloud particle size (middle) also in these column integrated values: The dayside (orange) shows a distinctly 
Ch. Helling et al.: Mineral cloud and hydrocarbon haze particles in the atmosphere of the hot Jupiter JWST target WASP-43b
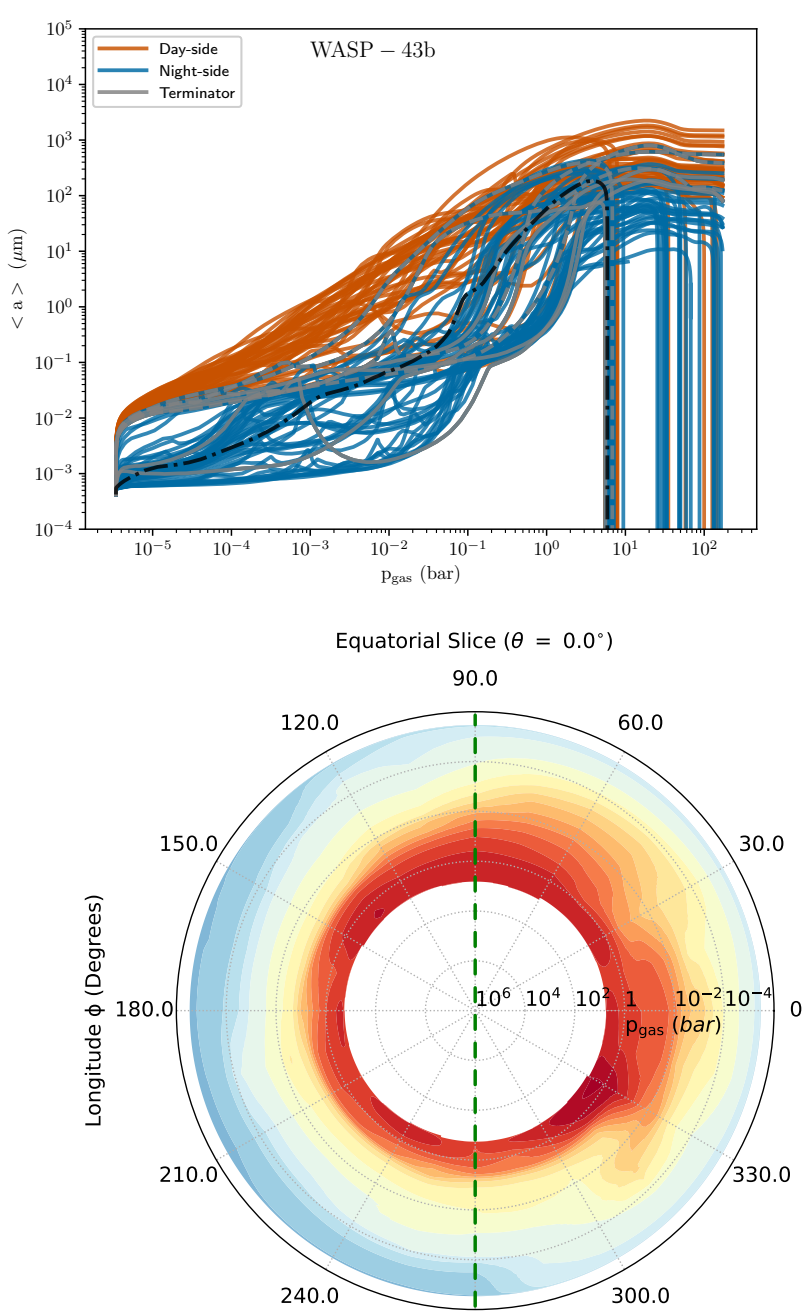

Terminator Slice $\left(\phi=90.0^{\circ}, 270.0^{\circ}\right)$

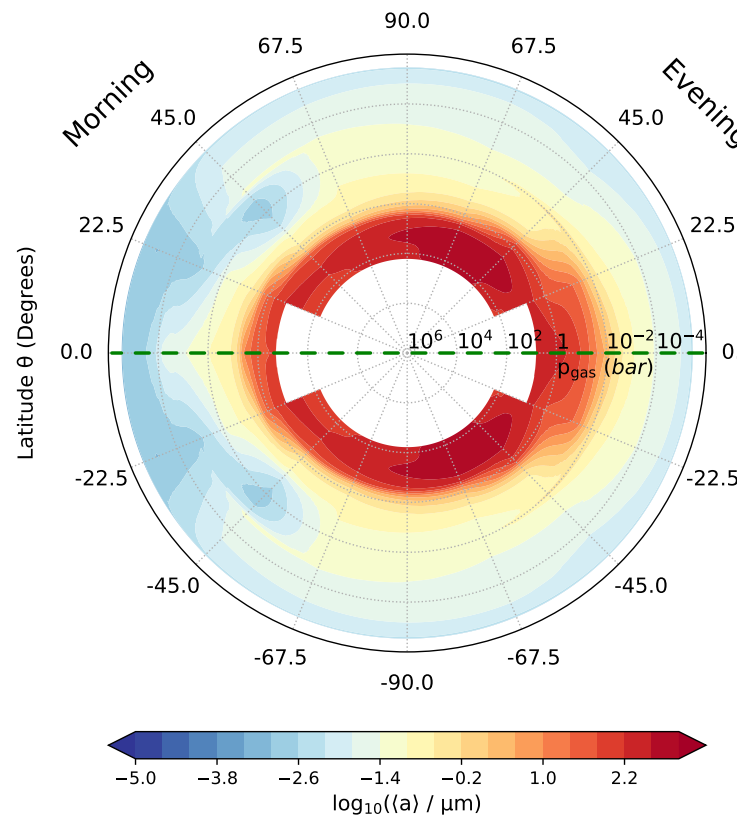

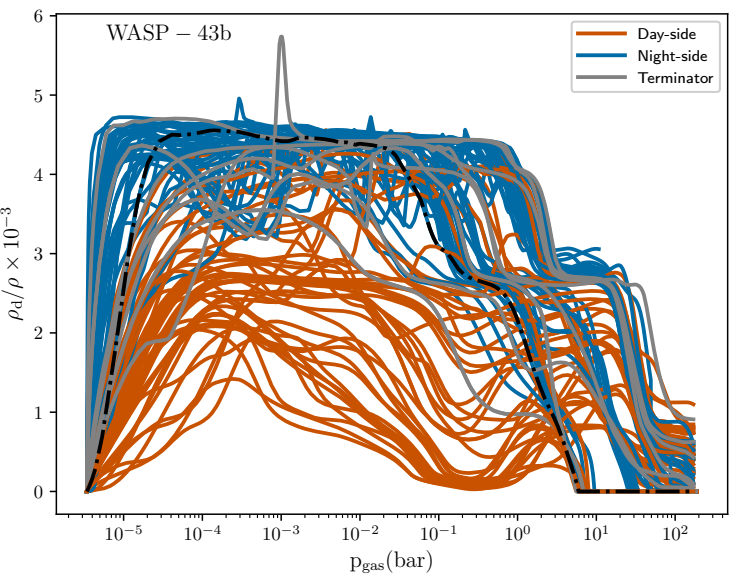

Equatorial Slice $\left(\theta=0.0^{\circ}\right)$

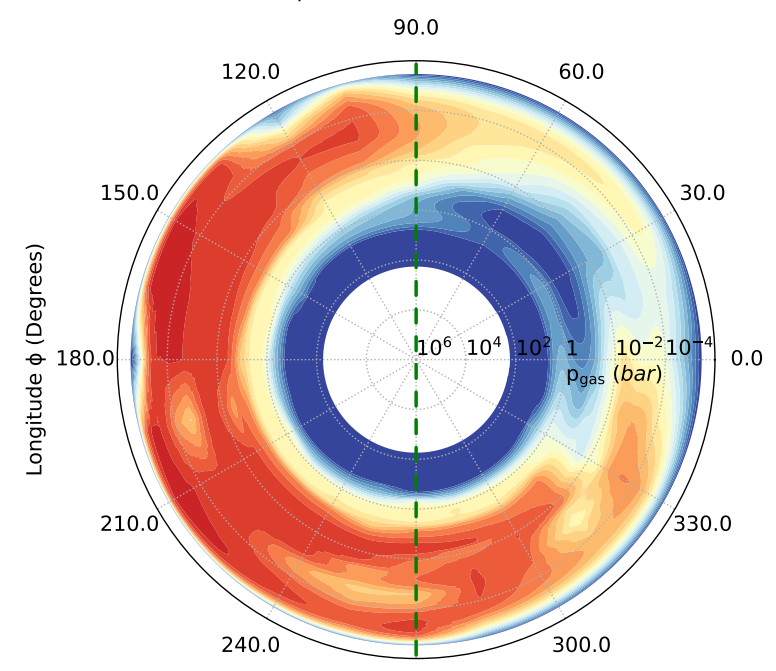

Terminator Slice $\left(\phi=90.0^{\circ}, 270.0^{\circ}\right)$

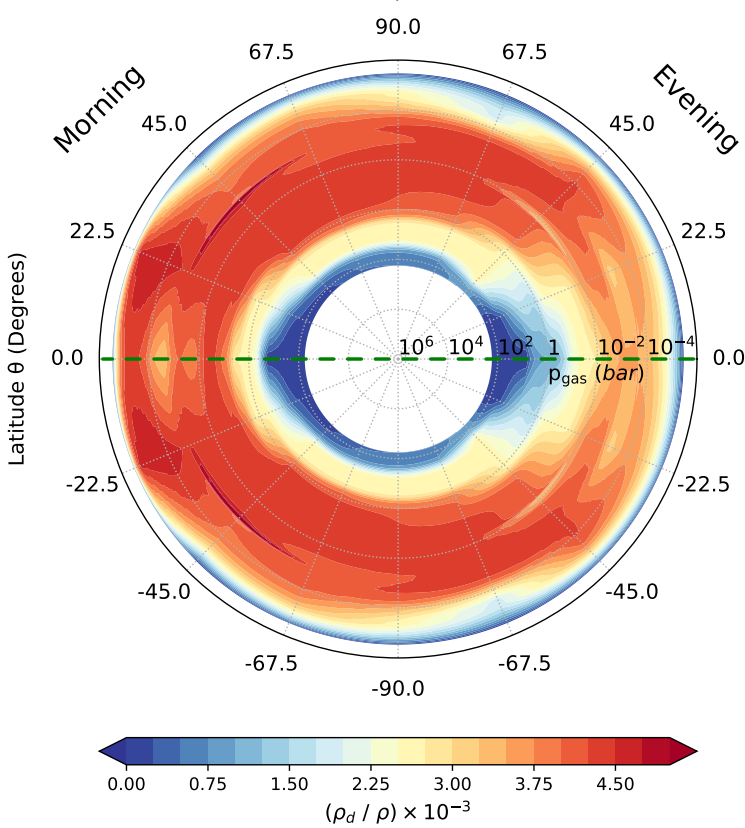

Fig. 6. Left: mean cloud particle size $\langle a\rangle[\mu \mathrm{m}]$. Right: cloud particle load in terms of dust-to-gas mass ratios, $\rho_{\mathrm{d}} / \rho_{\text {gas }}\left[10^{-3}\right]$. Top: for all 1D trajectories. The nightside profiles are shown in blue, the dayside profiles in orange, the terminator profiles in grey. Middle: $2 \mathrm{D}$ cut visualising the spatial location in the equatorial plane. Bottom: 2D cut along the terminator for a tidally locked WASP-43b. 

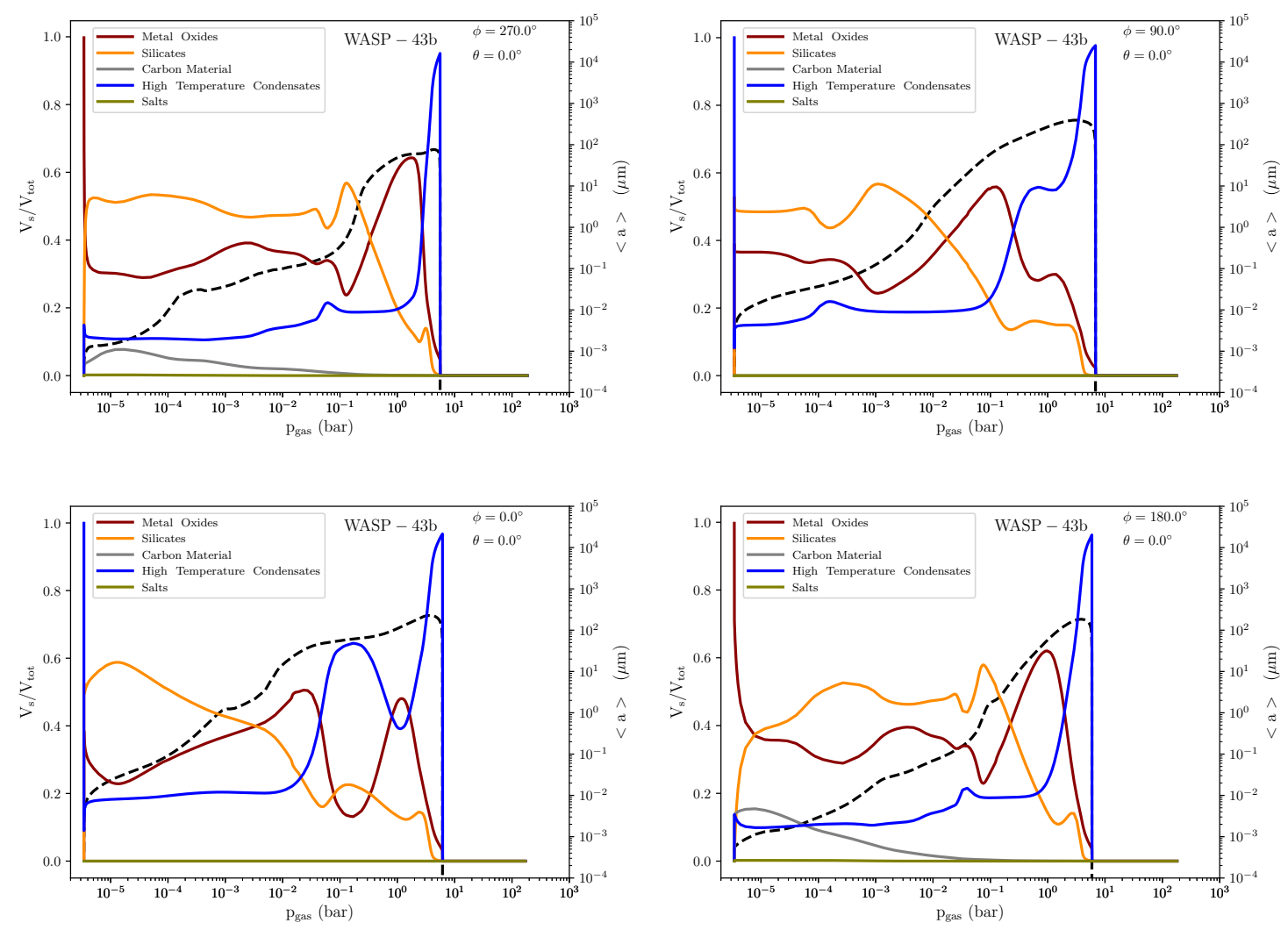

Fig. 7. Cloud particle material composition grouped into five categories for the morning terminator (top left), evening terminator (top right), sub-stellar point (bottom left), anti-stellar point (bottom right).

lower column integrated seed formation rate than the nightside which corresponds to distinctly larger average mean particle sizes on the dayside compared to the nightside. The terminator profiles are not included in Fig. 8.

Figure 8 (bottom) shows the column integrated values for the material composition $\left(\int_{\mathrm{zmin}}^{\mathrm{zmax}}\left(V_{\mathrm{s}}(z) / V_{\mathrm{tot}}(z)\right) \mathrm{d} z\right)$ for the substellar and the anti-stellar point and the two equatorial terminator points. These values demonstrate the contribution of the individual material groups to the composition of the whole cloud at this longitudes and latitudes. It suggests that the mineral clouds that form on WASP-43b are mainly made of silicates, metal oxides and high-temperature condensates. Salts like $\mathrm{KCl}[\mathrm{s}]$ play a less important role in the frame of the model used here. These integrated values do, however, not provide much insight into cloud formation in particular pressure regimes like for example the low-pressure regime that is tested by transmission spectroscopy.

\section{Gas phase composition in chemical equilibrium}

The atmospheric composition on WASP-43b is expected to be that of a warm, dense planet where cloud formation has affected the abundance of 11 elements $(\mathrm{Mg}, \mathrm{Si}, \mathrm{Ti}, \mathrm{O}, \mathrm{Fe}, \mathrm{Al}, \mathrm{Ca}, \mathrm{S}$, $\mathrm{C} \mathrm{K}, \mathrm{Cl}$ ) in a hydrogen-dominated atmosphere. Consequently, $\mathrm{H}_{2}$ is the most abundant gas-phase species inside the collision dominated part of the atmosphere of WASP-43b. This can change for the outermost, more diluted atmospheric layers where external high-energy radiation may cause $\mathrm{H}_{2}$ to photoionise similar to brown dwarfs (Rodríguez-Barrera et al. 2018). Brown dwarfs do form a shell of ionised gas (an ionosphere) in these photo-dissociation regions. $\mathrm{CO}$ appears as the most abundant molecule with $\mathrm{H}_{2} \mathrm{O}$ becoming more abundant only in the uppermost, low-pressure atmosphere $p_{\text {gas }}<10^{-4}$ bar on the nightside.
The abundance hierarchy of the 3rd and 4th most abundant gas species depends considerably on the local thermodynamic conditions (Fig. B.3). The equatorial evening terminator (longitude $\phi=90^{\circ}$ ) shows $\mathrm{CO}_{2}$ and $\mathrm{SiO}$ as the next most abundant gas species in chemical equilibrium. The equatorial morning terminator (longitude $\phi=270^{\circ}$ ) has considerably more $\mathrm{CH}_{4}$ than $\mathrm{CO}_{2}$ throughout. The substellar and the anti-stellar point show this shift in $\mathrm{SiO} / \mathrm{CH}_{4} / \mathrm{CO}$ abundances more clearly. On the dayside, $\mathrm{H}_{2} \mathrm{O}$ appears with a number density comparable to $\mathrm{CO}$ for $p_{\text {gas }}<0.1$ bar. Small carbon $\left(\mathrm{C}, \mathrm{C}_{2}\right)$ and hydrocarbon $(\mathrm{CH}$, $\left.\mathrm{CN}, \mathrm{C}_{2} \mathrm{H}_{2}, \mathrm{HCN}\right)$ molecules pertain their abundance hierarchy in contrast to the changing abundance of $\mathrm{CH}_{4}$ for the four profiles studied in more detail in Fig. B.3. Section 6 will assess their abundances under the effect of an external radiation field and vertical advection.

\subsection{Mean molecular weight and atmospheric scale height}

Complex models require physically justified assumptions. One of them is the mean molecular weight which allows an easy conversion of the total gas pressure into the total gas density applying the ideal gas law. Being able to assume a constant mean molecular weight when running an 3D GCM is computationally advantageous. We demonstrate here that this assumption is justified for the collision dominated part of the atmosphere of WASP-43b and we demonstrate it's effect on the hydrostatic atmospheric scale height. This will change where processes occur that for example photoionise $\mathrm{H}_{2}$, hence in the maybe molecular-dynamics dominated upper atmosphere where mass-loss mechanisms may take effect.

Based on our chemical equilibrium calculations that are affected by the depletion and enrichment of elements due to 

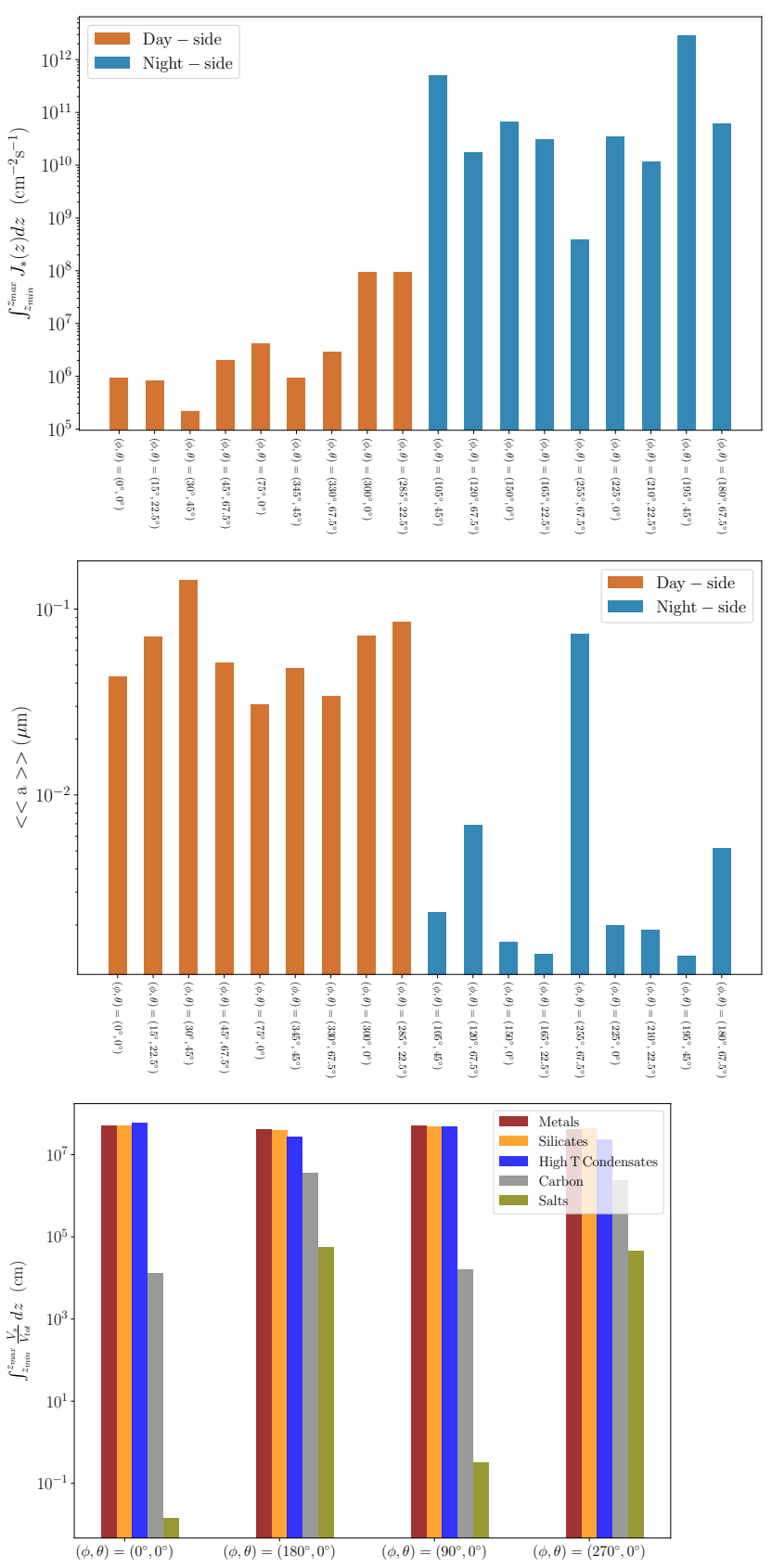

Fig. 8. Column integrated cloud properties for WASP-43b for 18 selected 1D profiles. Top: nucleation rate shown $\int_{z \min }^{z \max } J_{*}(z) \mathrm{d} z$ $\left[\mathrm{cm}^{-2} \mathrm{~s}^{-1}\right]$ with $J_{*}=\sum_{\mathrm{s}} J_{\mathrm{s}} \quad\left(s=\mathrm{TiO}_{2}, \quad \mathrm{SiO}, \mathrm{C}, \mathrm{KCl}\right)$. Middle: $\langle\langle a\rangle\rangle=\left(\int_{z \min }^{\mathrm{zmax}} n_{\mathrm{d}}(z)\langle a(z)\rangle \mathrm{d} z\right) /\left(\int_{\mathrm{zmin}}^{\mathrm{zmax}} n_{\mathrm{d}}(z) \mathrm{d} z\right)[\mu \mathrm{m}]$ with $n_{\mathrm{d}}(z)\left[\mathrm{cm}^{-3}\right]$ the local cloud particle number density. Bottom: material volume fraction: $\int_{\text {zmin }}^{\text {zmax }}\left(V_{\mathrm{s}}(z) / V_{\text {tot }}(z)\right) \mathrm{d} z$.

cloud formation (Fig. B.3, top) and based on the 1D ( $T_{\text {gas }}$, $\left.p_{\text {gas }}\right)$-profiles extracted from the Parmentier-3D GCM, the mean molecular weight is approximately constant $(\mu=2.31-2.3185)$ around the solar value of an $\mathrm{H}_{2}$-dominated atmosphere of $\mu=2.316$ (Fig. 9, top). Our photochemical simulations also show that the mean molecular weight is almost constant below the pressure range of $10^{-6}$ bar (i.e. GCM calculation range), above which it decreases except for the nightside as $\mathrm{H}$ becomes the dominant species due to the photodissociation of $\mathrm{H}_{2}$ (see Fig. 11). The nightside tends towards the higher values while the dayside tends towards the lower value in this range.
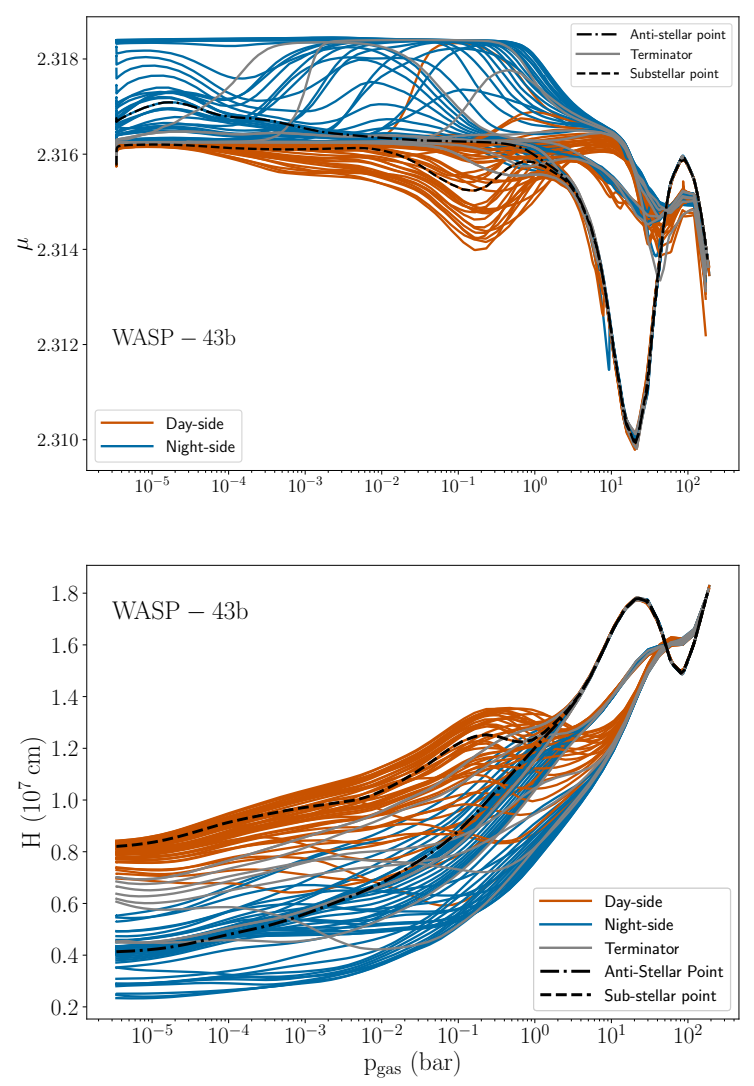

Fig. 9. Top: variations in the mean molecular weight, $\mu$ [amu], around the canonical value for a $\mathrm{H}_{2} / \mathrm{He}$ gas of $\mu=2.316$. Bottom: the resulting change in the atmospheric pressure scale height, $H\left[10^{7} \mathrm{~cm}\right]$, changes by $\Delta H \approx 6 \times 10^{6} \mathrm{~cm}$ from the day- to the nightside at $p_{\text {gas }}<0.5$ bar.

Casting this in terms of the hydrostatic pressure scale height, $H=k T_{\text {gas }} /\left(\mu m_{\mathrm{H}} g\right)$ (Fig. 9, bottom), the large temperature differences result in a change of the scale height of $H=2.5-8.5 \times$ $10^{6} \mathrm{~cm}$ in the upper, low pressure part of the atmosphere. The day-night-difference of $\Delta H \approx 6 \times 10^{6} \mathrm{~cm}(6000 \mathrm{~km})$ prevails throughout the atmospheric profiles for pressures $p_{\text {gas }}<0.5$ bar.

\subsection{The carbon-to-oxygen ratio}

Mineral ratios like $\mathrm{Si} / \mathrm{O}, \mathrm{Mg} / \mathrm{O}$ and the carbon-to-oxygen ratio, $\mathrm{C} / \mathrm{O}$, may enable linking observations to evolutionary stages of exoplanets. The challenge herewith is that observations have so far only provides snippets of the atmosphere spectrum, that is information about limited wavelength intervals. This limitation in combination with a limited range of molecular absorbers included in retrieval codes has led to suggestions of $\mathrm{C} / \mathrm{O}>1$, i.e. carbon-rich exoplanet atmospheres. The carbon abundance can only exceed the oxygen abundance if either carbon is produced (like in AGB stars) or inserted otherwise into the system (for example, meteoritic influx), or if the oxygen is simply reduced below the original carbon-abundance level. The latter might occur if the planet already starts out with a primordial atmosphere that is oxygen-depleted due to the formation of $\mathrm{H}_{2} \mathrm{O} / \mathrm{CO}$ ices inside the planet-forming disc. The accretion of the gaseous envelope would then occur from this oxygen-depleted gas and further processing of the atmospheric gas into clouds can then lead to a $\mathrm{C} / \mathrm{O}>1$ (Helling et al. 2014).

Figure 10 details $\mathrm{C} / \mathrm{O}$ for the 97 1D profiles that we have investigated for the atmosphere of WASP-43b. C/O traces the location of the cloud in pressure space: a sharp increase defines 

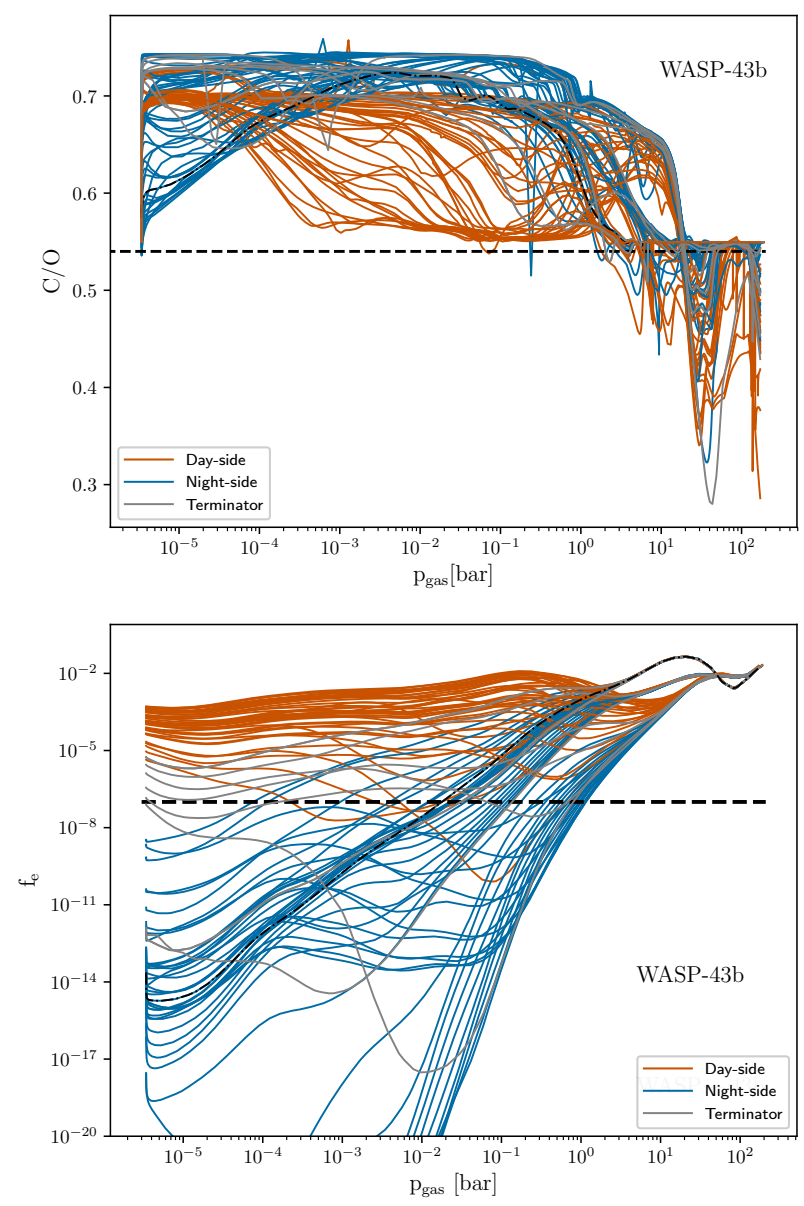

Fig. 10. Top: carbon-to-oxygen ratio, $\mathrm{C} / \mathrm{O}$ (dashed horizontal line: solar $\mathrm{C} / \mathrm{O}$ ). Bottom: degree of ionisation, $f_{\mathrm{e}}=p_{\text {gas,tot }} / p_{\mathrm{e}}$ (dashed horizontal line: $f_{\mathrm{e}}=10^{7}$ as a threshold value for plasma behaviour). C/O and $f_{\mathrm{e}}$ are shown for all 1D profiles studied here for the atmosphere of WASP-43b. The nightside profiles are shown in blue, the dayside profiles in orange, the terminator profiles in grey.

the cloud top which is where oxygen is effectively stored in cloud particles. The detailed shape of the $\mathrm{C} / \mathrm{O}$ curves depend on the details of the atmospheric temperature and pressure but it is clear that depletion pushes $\mathrm{C} / \mathrm{O}$ to values of $\approx 0.74$. The trend reverses in deeper atmospheric layers where high temperature cause oxygen-carrying solids to evaporate. An enrichment of oxygen results and, hence, $\mathrm{C} / \mathrm{O}$ decreases to values as low as 0.3 . $\mathrm{C} / \mathrm{O}$ values for WASP-43b do vary throughout the atmosphere and between the day- and the nightside.

\subsection{The ionised fraction of the atmospheric gas on WASP $43 b$}

WASP-43b is a giant gas planet that orbits its host star rather closely at a distance of $a \approx 00153 \mathrm{au}$ in 0.813 days, but its host star is comparably cool compared to ultra-hot Jupiters like WASP-18b or HAT-P-7b. The degree of ionisation, $f_{\mathrm{e}}=p_{\mathrm{e}} /\left(p_{\mathrm{gas}}\right.$ ( $p_{\mathrm{e}}-$ electron pressure, $p_{\text {gas }}-$ the total gas pressure), is low on the day- and on the nightside (Fig. 10, bottom). The atmosphere on the dayside can be considered as partially ionised as $f_{\mathrm{e}} \approx 10^{-3.5}>10^{-7}$. While $f_{\mathrm{e}} \approx 10^{-7}$ has been postulated as threshold for possible plasma behaviour in a gas, follow-up studies are required to quantify this for WASP-43b as outlined in Rodríguez-Barrera et al. (2015). The dominating electron donors are $\mathrm{K}^{+}$and $\mathrm{Na}^{+}$, followed by $\mathrm{Ca}^{+}$. This is very much in line with results obtained for brown dwarfs (see Fig. 3 in RodríguezBarrera et al. 2015). $\mathrm{H}^{-}$appears as the third most abundant ion for WASP-43b (Fig. B.4).

\section{The $\mathrm{C} / \mathrm{H} / \mathrm{O} / \mathrm{N}$ molecule abundances under the effect of mixing and UV radiation and the formation of hydrocarbon hazes on WASP-43b}

We wish to explore the effect of photochemistry on the cloud particle formation in the atmosphere of WASP-43b. We explore the formation of hydrocarbon hazes as result of hydrocarbon monomers coagulating to larger hydrocarbon aggregate structures from an oxygen-rich $(\mathrm{C} / \mathrm{O}<1)$ atmospheric gas in addition to the formation of mineral cloud particles. In a non-irradiated oxygen-rich atmosphere $(\mathrm{C} / \mathrm{O}<1)$, most of the carbon would be locked up in $\mathrm{CO}$ in such a high-temperature atmosphere unless the strong $\mathrm{CO}$ bond is destroyed. $\mathrm{CH}_{4}$ becomes the most dominant one in low-temperature atmospheres. We perform kinetic $\mathrm{C} / \mathrm{H} / \mathrm{O} / \mathrm{N}$ chemistry calculations for the two terminators $\left(\phi=90^{\circ}, 270^{\circ}\right)$, the substellar $\left(\phi=0^{\circ}\right)$ and the anti-stellar point $\left(\phi=180^{\circ}\right)$ at the equator $\left(\theta=0^{\circ}\right)$. First, we discuss the photochemical $\mathrm{C} / \mathrm{H} / \mathrm{O} / \mathrm{N}$ gas-phase results (Sect. 6.1). Section 6.2 discusses our results for the hydrocarbon haze at the three selected atmospheric profiles. We note, however, that the profiles of temperature and eddy diffusion coefficient have been extrapolated outside the GCM calculation domains in order to capture the full potential of hydrocarbon haze formation in the upper and lower atmospheres (Fig. B.1). The photochemical calculation domain extends from 1000 bar to the pressure at the highest altitude grid, which is different for each calculation and also varies during the calculation due to the change in mean molecular weight in the upper atmosphere because of the molecular hydrogen decomposition. The calculation region to the lower atmosphere is very important to simulate the atmospheric region where the pressure is high enough for the thermochemistry to dominate because the chemistry in the lower atmosphere greatly affects that in the upper atmosphere through the vertical mixing. We note here that a deeper inner boundary of the 3D GCM simulation may affect the atmosphere structure (Carone et al. 2020), but this has not been considered in our paper. While the temperatures in the upper atmosphere are extrapolated isothermally, those in the lower atmosphere are extrapolated with the same temperature-pressure gradient. Eddy diffusion coefficients are extrapolated with the values at the upper and lower boundaries of the mineral cloud calculation region. The complete $\left(T_{\mathrm{gas}}, p_{\mathrm{gas}}\right.$, $\left.K_{\mathrm{zz}}\right)$ information for these profiles are provided in Fig. B.1.

\subsection{Kinetic, photochemical gas-phase results for $\mathrm{C} / \mathrm{H} / \mathrm{O} / \mathrm{N}$ chemistry}

In Fig. 11, we show the results of our photochemical calculations for (a) morning terminator $\left(\phi=270^{\circ}\right)$, (b) evening terminator $\left(\phi=90^{\circ}\right)$, (c) sub-stellar point $\left(\phi=0^{\circ}\right)$ and (d) anti-stellar point $\left(\phi=180^{\circ}\right)$. Among the four atmospheric 1D profiles probed here, the anti-stellar point $\left(\phi=180^{\circ},(\mathrm{d})\right)$ is the simplest due to the absence of photochemistry. In the lower atmosphere $\left(p_{\text {gas }} \gtrsim\right.$ 1 bar), the volume mixing ratio of each species (solid line) is consistent with its thermochemical equilibrium abundance (dashed line). This is because the timescales of thermochemical reactions are shorter than eddy diffusion transport timescales due to the high temperatures and pressures in the lower atmosphere. Many species such as $\mathrm{HCN}, \mathrm{C}_{2} \mathrm{H}_{2}$ and $\mathrm{OH}$ are created because of their 


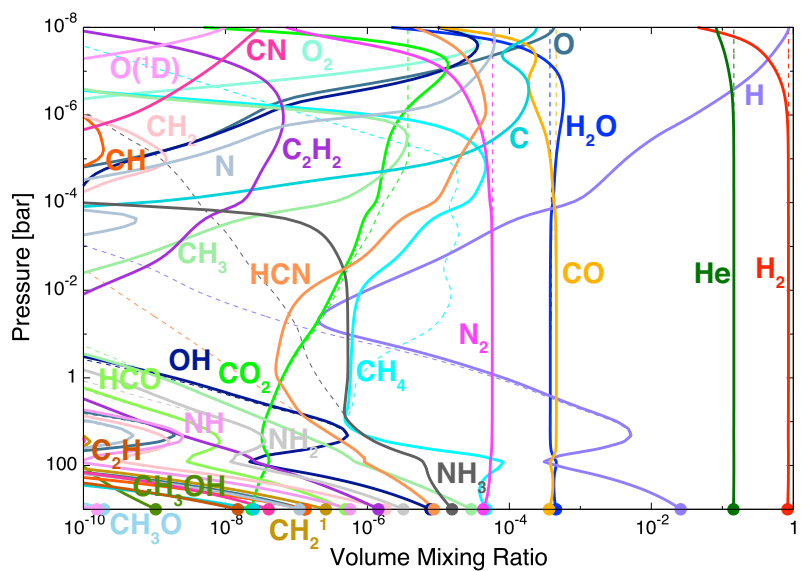

(a) Morning terminator $\left(\phi=270^{\circ}\right)$

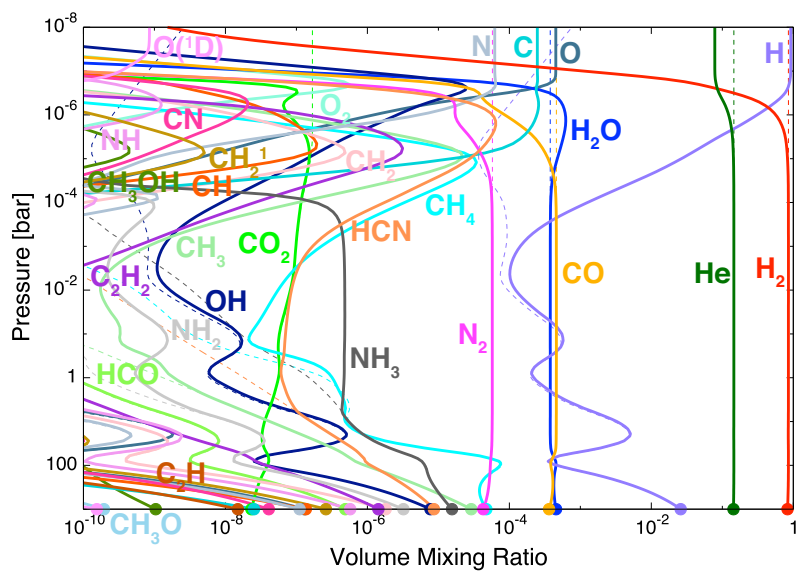

(c) Sub-stellar point $\left(\phi=0^{\circ}\right)$

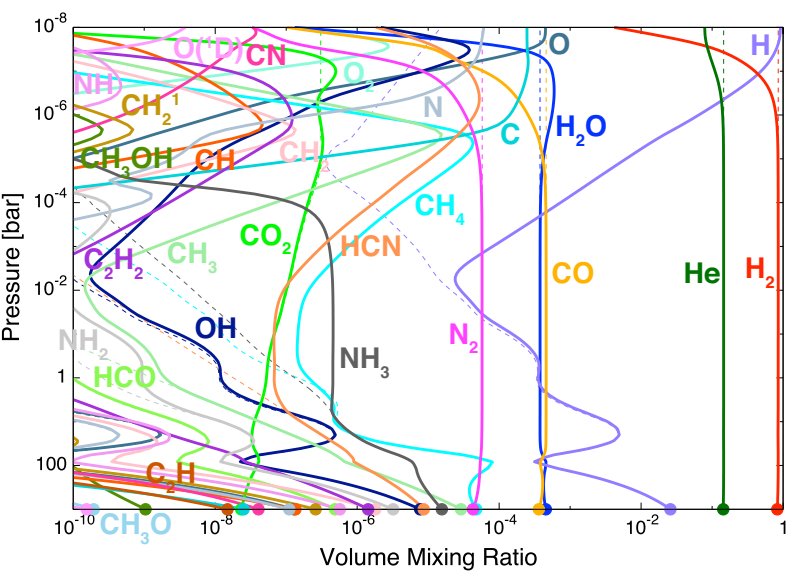

(b) Evening terminator $\left(\phi=90^{\circ}\right)$

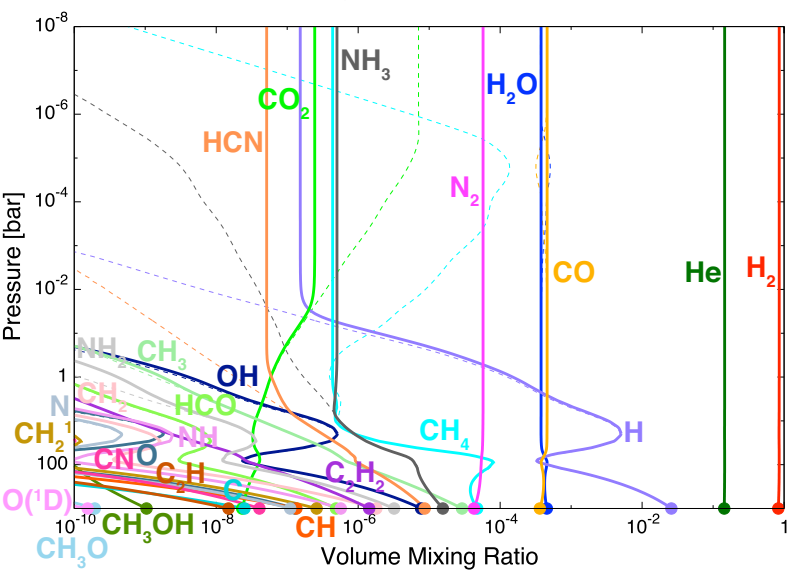

(d) Anti-stellar point $\left(\phi=180^{\circ}\right)$

Fig. 11. Kinetic photochemical equilibrium $\mathrm{C} / \mathrm{H} / \mathrm{O} / \mathrm{N}$ gas-phase results (solid line) for the 1D WASP-43b profiles at $(a)$ the morning terminator $\left(\phi=270^{\circ}\right),(b)$ the evening terminator $\left(\phi=90^{\circ}\right),(c)$ the sub-stellar point $\left(\phi=0^{\circ}\right)$ and $(d)$ the anti-stellar point $\left(\phi=180^{\circ}\right)$. The filled circles represent the thermochemical equilibrium values at the lower boundary. For reference, the dashed lines show the abundances in thermochemical equilibrium, where the eddy diffusion transport is also ignored.

stability at high temperatures. On the other hand, the upper atmosphere is dominated by eddy diffusion mixing as can be seen for some species that their abundances are quenched at certain pressure levels; $\sim 10^{-2}$ bar for $\mathrm{CO}_{2}$ and $\mathrm{H}, \sim 1$ bar for $\mathrm{CH}_{4}$ and $\mathrm{HCN}$ and $\sim 10$ bar for $\mathrm{NH}_{3}$.

The other three cases with $\mathrm{C} / \mathrm{H} / \mathrm{O} / \mathrm{N}$ photochemistry are more complex, but the chemistry in the lower atmosphere is similar since the $\left(T_{\text {gas }}, p_{\text {gas }}\right)$-profiles at such a high pressure range is almost the same (see Fig. B.1). Also, it is the same as the case of (d) anti-stellar point $\left(\phi=180^{\circ}\right)$ that the middle atmosphere ( 1 bar $\lesssim p_{\text {gas }} \lesssim 10^{-4}$ bar) is dominated by eddy diffusion mixing. The upper atmosphere ( $p_{\text {gas }} \lesssim 10^{-4}$ bar) is, however, governed by photochemistry. Many species, the abundances of which in thermochemical equilibrium are quite low, such as $\mathrm{H}, \mathrm{O}, \mathrm{C}, \mathrm{HCN}$, $\mathrm{N}, \mathrm{OH}, \mathrm{CH}_{3}, \mathrm{C}_{2} \mathrm{H}_{2}, \mathrm{CH}_{2}, \mathrm{O}_{2}, \mathrm{CH}, \mathrm{CN}, \mathrm{CH}_{2}^{1}, \mathrm{NH}, \mathrm{O}\left({ }^{1} \mathrm{D}\right)$ and $\mathrm{CH}_{3} \mathrm{OH}$ are produced by photochemical reactions. As for the haze precursors (i.e. $\mathrm{CH}_{4}, \mathrm{HCN}$ and $\mathrm{C}_{2} \mathrm{H}_{2}$ ), they are all created through the photochemistry in the upper atmosphere while $\mathrm{HCN}$ and $\mathrm{C}_{2} \mathrm{H}_{2}$ in the lower atmosphere are created thorough the thermochemical reactions.

Compared to the cases of the two terminators, where only the one side facing to the host star is irradiated, sub-stellar point $\left(\phi=0^{\circ}\right.$, (c) ) receives twice the amount of the incoming stellar photon flux (see Sect. 2), resulting in more efficient photochemistry. This effect can be seen at, for example, the slightly higher pressure levels (lower altitudes) of the dissociation of $\mathrm{H}_{2}, \mathrm{H}_{2} \mathrm{O}, \mathrm{CO}, \mathrm{CH}_{4}, \mathrm{HCN}$ and $\mathrm{C}_{2} \mathrm{H}_{2}$ and the existence of photochemically-produced species mentioned above at somewhat higher pressure levels (lower altitudes). As for the comparison between the two terminators, the only differences in the calculation settings are the assumed profiles of temperaturepressure and eddy diffusion (see Fig. B.1) and the amount of the incoming photon flux is the same. The effect of the higher temperatures for the evening terminator can be seen at the larger number of the photochemically-created species in the upper atmosphere (because high temperatures also favour these high-energy species).

The resultant sum of the photodissociation rates of the three haze precursors, which is assumed to be the monomer production rate in the particle growth simulations, integrated throughout the atmospheres are $2.61 \times 10^{-11}, 8.19 \times 10^{-11}$ and $2.48 \times 10^{-11}\left[\mathrm{~g} \mathrm{~cm}^{-2} \mathrm{~s}^{-1}\right] \quad\left(6.23 \times 10^{9}, 1.95 \times 10^{10}\right.$ and $\left.5.91 \times 10^{9} \mathrm{~cm}^{-2} \mathrm{~s}^{-1}\right)$ for (a) morning terminator $\left(\phi=270^{\circ}\right)$, (b) evening terminator $\left(\phi=90^{\circ}\right)$, (c) sub-stellar point $\left(\phi=0^{\circ}\right)$ and (d) anti-stellar point $\left(\phi=180^{\circ}\right)$, respectively. Unlike the case of the cool ( $~ 500 \mathrm{~K})$ planets (Kawashima \& Ikoma 2019), we find that $\mathrm{HCN}$ rather than $\mathrm{C}_{2} \mathrm{H}_{2}$ contributes most to the haze formation among the three precursors because of its stability at high temperatures for all the three longitude points. Here we note the link to a possible lighting-induced boost of $\mathrm{HCN}$ in cloudy 
exoplanets (Rimmer \& Helling 2016; Hodosán et al. 2016). The monomer production rate for the sub-stellar point is more than twice the values of the terminators. We find that this is because of the decreased abundances of the major-photon absorbers of $\mathrm{H}_{2}$ and $\mathrm{CO}$ in the upper atmosphere such that $\mathrm{HCN}$ can use higher ratio of the incoming photons for its photodissociation for the case of the sub-stellar point. The production rates of the two terminators are almost similar, which is reasonable since the incoming stellar photon flux is exactly the same.

The atmospheric chemistry of WASP-43b was also explored by Mendonça et al. (2018a) and Venot et al. (2020). Mendonça et al. (2018a) simulated the 3D profile of the atmospheric composition considering the horizontal and vertical transport with the use of the global circulation model THOR (Mendonça et al. 2016), but without photochemistry. Our temperature-profiles of the sub- and anti-stellar points and morning and evening terminators are similar to their cases of $G=0.5$, where $G$ is their greenhouse parameter. Comparing the vertical profiles of the volume mixing ratios of the four molecules they presented, namely $\mathrm{H}_{2} \mathrm{O}, \mathrm{CO}, \mathrm{CH}_{4}$ and $\mathrm{CO}_{2}$ at the four longitude points with ours, the chemistry of the lower atmospheres is almost similar although we ignore horizontal transport. This is because the temperature profiles in the lower atmosphere are basically the same along the latitude and thus the volume mixing ratios at thermochemical equilibrium state are similar anyway. We note that they found that the timescale of the horizontal transport in the longitude direction is always shorter than that of the vertical transport while that of the horizontal transport in the latitude direction is comparable to the vertical one. However, since they did not include photochemistry while we have ignored horizontal transport, the profiles in the upper atmosphere are different. The abundance of $\mathrm{CH}_{4}$ is much larger due to the molecular photodissociation and all the four molecules are dissociated in the very upper atmosphere in our case.

Venot et al. (2020) studied the chemical structure of WASP$43 \mathrm{~b}$ considering the effect of horizontal circulation with the use of their pseudo-2D model in addition to the 1D model. Our VMR profiles of the major species such as $\mathrm{H}_{2} \mathrm{O}, \mathrm{CO}, \mathrm{NH}_{3}, \mathrm{~N}_{2}, \mathrm{HCN}$ and $\mathrm{CO}_{2}$, are basically similar to their $1 \mathrm{D}$ results while the dayside profile of $\mathrm{CH}_{4}$ is different probably due to the differences of the assumed stellar UV spectrum and chemical network in addition to the different assumed profiles of temperature and eddy diffusion coefficient. They demonstrated that the horizontal circulation reduces the gradient of chemical composition along the longitude especially in the pressure range of $10^{-4}-1$ bar. The abundance profiles for the two terminators and the anti-stellar point presented here should be somewhat homogenised to that of the dayside due to the horizontal quenching effect we have ignored.

\subsection{Hydro-carbon haze formation}

Applying the monomer production rate derived in Sect. 6.1, the hydrocarbon cloud particles are now calculated from a coagulation model simulating the growth by particle-particle coagulation processes under the effect of gravity and vertical mixing due to eddy diffusion. Figure 12 shows the calculated vertical profiles of haze properties for evening terminator $\left(\phi=90^{\circ}\right.$, top), sub-stellar point ( $\phi=0^{\circ}$, middle) and morning terminator $\left(\phi=270^{\circ}\right.$, bottom). The resultant profiles of the haze particle are basically similar because of the only a factor of differences in the monomer production rate (see Kawashima \& Ikoma 2018, for the dependence on monomer production rate). The particles
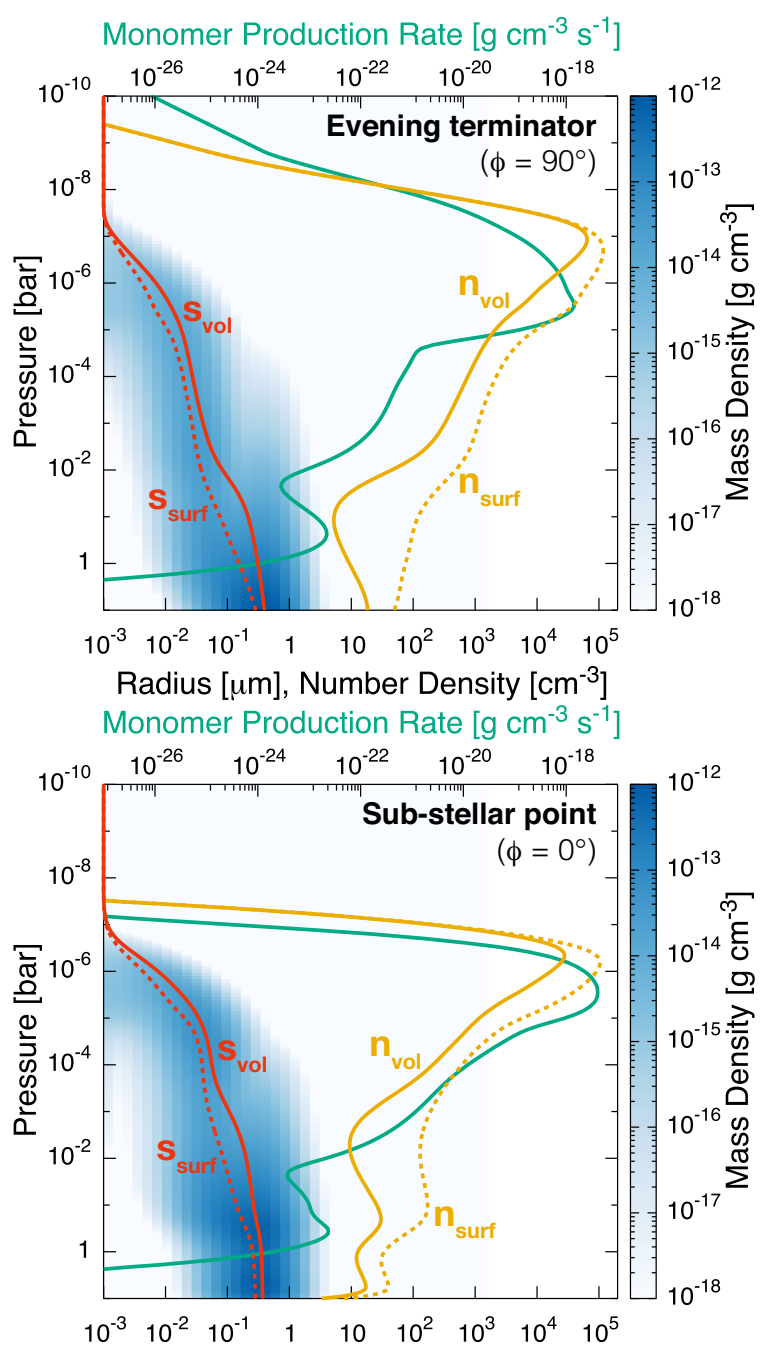

Radius [um], Number Density $\left[\mathrm{cm}^{-3}\right]$

Monomer Production Rate $\left[\mathrm{g} \mathrm{cm}^{-3} \mathrm{~s}^{-1}\right]$

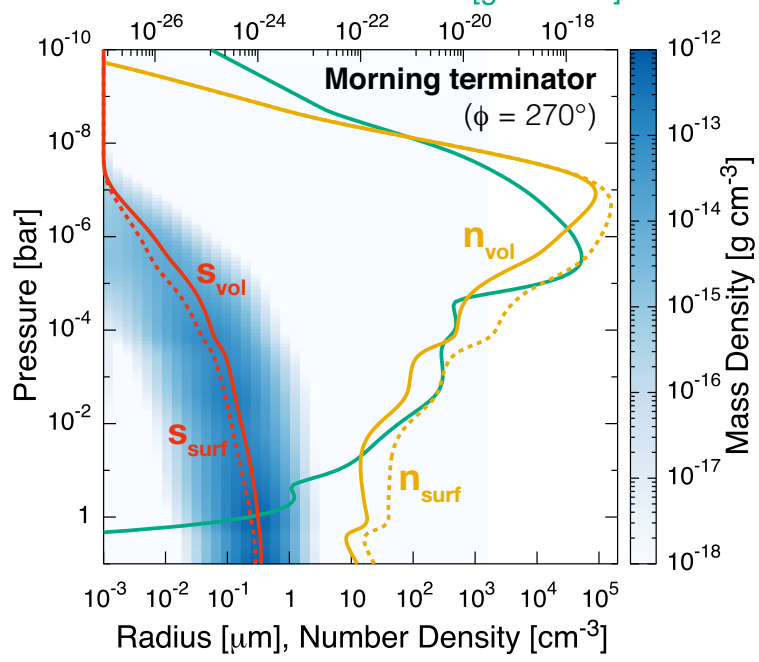

Fig. 12. Haze particles profiles for the $1 D$ WASP-43b profiles at the evening terminator $\left(\phi=90^{\circ}\right.$, top $)$, the sub-stellar point $\left(\phi=0^{\circ}\right.$, middle) and the morning terminator $\left(\phi=270^{\circ}\right.$, bottom); volume average radius $s_{\mathrm{vol}}$ (solid red) and number density $n_{\mathrm{vol}}$ (solid orange) and the surface average radius $s_{\text {surf }}$ (dotted red) and number density $n_{\text {surf }}$ (dotted orange), along with that of the monomer mass production rate (solid green). See Kawashima \& Ikoma (2018) for the definition of each quantity. 
start to grow at $p_{\text {gas }} \sim 10^{-6}$ bar and result in the average sizes of $\sim 3-4 \mu \mathrm{m}$ at the lower boundary of $p_{\text {gas }} \sim 10$ bar.

The monomer production rate profiles (green lines) have the peaks at certain pressures since the photodissociation rates are determined by both the number of available photons, which decreases with increasing pressures and the number of the molecules, which basically increases with increasing pressures. The second peaks can be seen at $p_{\text {gas }} \sim 1$ bar come from the increase of the $\mathrm{C}_{2} \mathrm{H}_{2}$ number density due to its stability at high temperatures. Compared to the two terminators, the case of the sub-stellar point has more concentrated monomer production profile because the photodissociation of the haze precursors begins to occur at higher pressure (lower altitude) but with the higher rate due to the intense incoming photon flux. This result in the more broader size distribution in this case, which can be seen as the slightly larger difference between the volume and surfaced average radii. Here we note that we have ignored the thermal decomposition due to the uncertainty of the thermal stability of haze, which can affect the particles in the high-temperature lower atmosphere.

\section{Two ensembles of chemically distinct cloud particles in oxygen-rich exoplanet atmospheres}

Mineral cloud particles may form by distinctly different processes than hydro-carbon cloud particles due to the presence of a radiation field in an (atmosphere) environment of changing density. The mineral cloud particles discussed here (Sect. 4) to form in the atmosphere of WASP-43b originate from an oxygenrich gas $(\mathrm{C} / \mathrm{O}<1)$ through gas-gas and gas-particle reactions from a highly supersaturated gas. The hydro-carbon cloud particles, also called "hydro-carbon haze", form under the influence of an UV radiation field by photo-chemical processes from the small hydro-carbon molecules like $\mathrm{CH}_{4}, \mathrm{C}_{2} \mathrm{H}_{2}$ and $\mathrm{HCN}$ (Sect. 6.2). The resulting monomers grow through particleparticle collisions (coagulation) to larger cloud particles in form of aggregates. The processes involved in the formation of the two cloud particle ensembles, mineral particles and hydrocarbon hazes, are distinctly different and we present here a study how this two cloud particle ensembles differ in their properties like nucleation rates, cloud particles sizes and cloud particle number densities.

\subsection{Mineral seed and hydro-carbon haze formation rates}

We present both cloud ensembles in Fig. 13 by comparing our results for mineral seed formation applying modified classical nucleation theory as described in Sect. 2 for the atmosphere of WASP 43b with a haze monomer production rate, which is defined as the sum of the photodissociation rates of the three haze precursor, $\mathrm{CH}_{4}, \mathrm{HCN}$ and $\mathrm{C}_{2} \mathrm{H}_{2}$, (this is the upper limit of the haze monomer production rate) by assuming a monomer size of $1 \mathrm{~nm}$ and its density of $1 \mathrm{~g} \mathrm{~cm}^{-3}$ giving a mass of $4.19 \times$ $10^{-21} \mathrm{~g}$ per $\mathrm{C} / \mathrm{H} / \mathrm{O} / \mathrm{N}$ haze monomer. The mineral seed particles have a material density of $4.23 \mathrm{~g} \mathrm{~cm}^{-3}$ for $\mathrm{TiO}_{2}, 2.64 \mathrm{~g} \mathrm{~cm}^{-3}$ for $\mathrm{SiO}$ and $1.98 \mathrm{~g} \mathrm{~cm}^{-3}$ for $\mathrm{KCl}$.

For photochemically driven gas-phase reactions, the hydrocarbon haze formation rate, which is defined as the sum of the photodissociation rates of the haze precursors, appears to be more efficient than mineral seed particles on the highly irradiated dayside of WASP-43b. The photodissociation rates of the haze precursors are almost the same for the three longitude points studied here because the sub-stellar point receives only
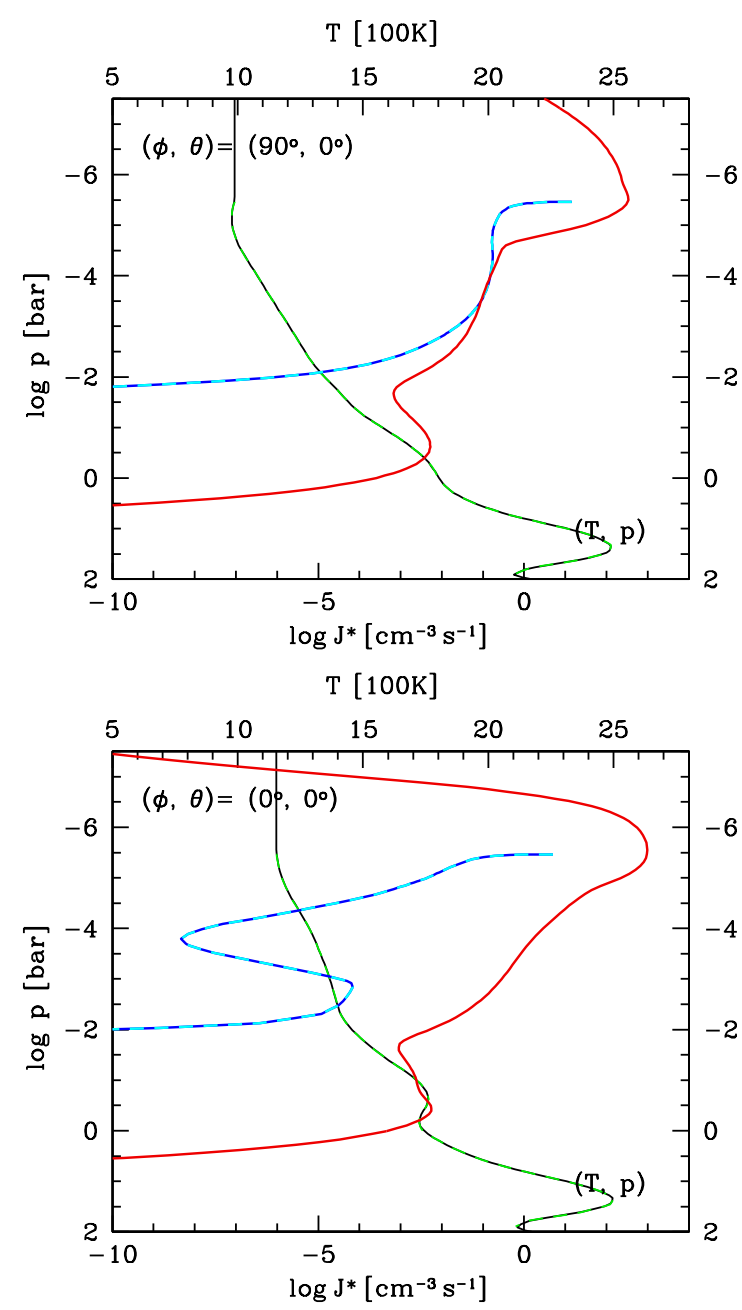

$\mathrm{T}[100 \mathrm{~K}]$

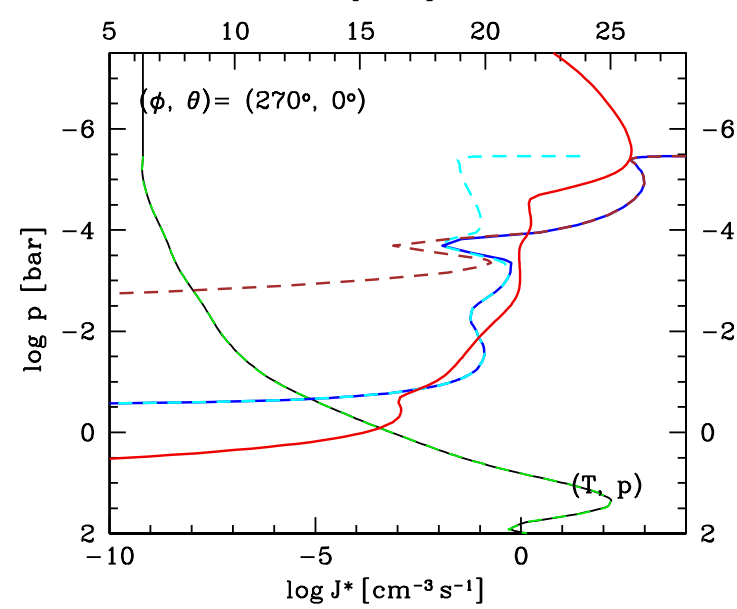

Fig. 13. Two cloud particle ensembles: the mineral seed formation rate (blue: total value of the mineral seed particles $\mathrm{TiO}_{2}$ (cyan dashed), $\mathrm{SiO}$ (brown dashed) and $\mathrm{KCl}$ (green, not occurring at equator)) and the hydrocarbon haze particles formation rate (red). The $\left(T_{\mathrm{gas}}, p_{\mathrm{gas}}\right)$ structure is shown in black (extrapolated) and green dashed (original $3 \mathrm{D}$ GCM).

two times higher UV irradiation compared to the two terminator points. Negligible amount of photochemical hazes will form on the nightside as no or only very small amounts of stellar photons are available. Horizontal circulation may be able to transport particles from the dayside to the nightside. 
The whole cloud extends throughout the whole computational domain of the utilised 1D trajectories (Figs. 2 and 6) because gravitational settling transports the cloud particles inwards. Gravitational settling and thermal stability in the case of mineral cloud particle, determine the geometrical cloud extension and hence, the inner cloud boundary. This is not yet been taken into account for the photochemical haze comparison as shown in Figs. 13 and 14 due to the uncertainty of the thermal stability of haze.

\subsection{Mineral particle and hydrocarbon haze number density and mean particle sizes}

As a consequence of the differences between the mineral and the hydrocarbon haze nucleation rate, the hydrocarbon number densities (green dashed line in Fig. 14) are larger than the mineral particles densities (green solid line) at the substellar point $\left((\phi, \theta)=\left(0^{\circ}, 0^{\circ}\right)\right)$ but also at the evening terminator $\left((\phi, \theta)=\left(90^{\circ}, 0^{\circ}\right)\right)$. With the local temperature decreasing by $\sim 500 \mathrm{~K}$ at the morning terminator $\left((\phi, \theta)=\left(270^{\circ}, 0^{\circ}\right)\right)$ compared to the substellar point, the mineral seed formation rate creates a larger abundance of mineral cloud particles at the morning terminator. The number of cloud particles has direct implications for the resulting (mean) sizes as a larger number provides more surface to condense on and hence, mineral cloud particles will remain smaller. This is why the morning terminator $\left((\phi, \theta)=\left(270^{\circ}, 0^{\circ}\right)\right)$ has smaller mineral cloud particles than the evening terminator $\left((\phi, \theta)=\left(90^{\circ}, 0^{\circ}\right)\right)$. This logic does not hold for the hydrocarbon haze particles which appear almost the similar sizes for all the three points because of the slight differences in the monomer production rates. This is because the mineral particle growth significantly depends on the surrounding bulk gas phase (and accelerates with increasing density) but for the case of the hydrocarbon haze, its formation is more governed by additional photochemical process.

Comparing model results by number hinges on an exact definition of the compared quantities. Figure A.1 (orange and brown lines) demonstrates the differences that result in the values of cloud particle sizes when derived as weighted average $\left(\langle a\rangle\right.$, Eq. (A.3), solid lines), as surface weighted average $\left(\langle a\rangle_{\mathrm{A}}\right.$, Eq. (A.6), dashed line) and as $\sqrt[3]{\left\langle a^{3}\right\rangle}$ (Eq. (A.8), dotted line) from our two different methods (moment equation vs. binning method). Figure 14 demonstrates that differences of 0.5 order of mag may occur.

Our comparison suggests that surface growth processes lead to larger particles in particular in the inner, denser atmospheric regions and that hydrocarbon cloud particles may remain smaller, more haze-like. The upper, low-pressure regime where $p_{\text {gas }}<10^{-4}$ bar is populated by an ensemble of small hydrocarbon haze particles of $<10^{-2.5} \mu \mathrm{m}$ and mineral particles of $>10^{-2} \mu \mathrm{m}$. Therefore, transmission spectra may be affected by two cloud particle populations in low density atmospheres regions where photochemistry is efficient but surface growth processes are not yet efficient because of the low gas-surface collision rates. However, a simple retrieval of particles sizes will not suffice to distinguish between the two cloud particle ensembles, in particular as it is degenerate with the particle number density of the opacity species.

\section{Discussion of observational implications}

Cloud particles tend to dominate the optical depth of an atmosphere such that a planet would appear with a larger radius
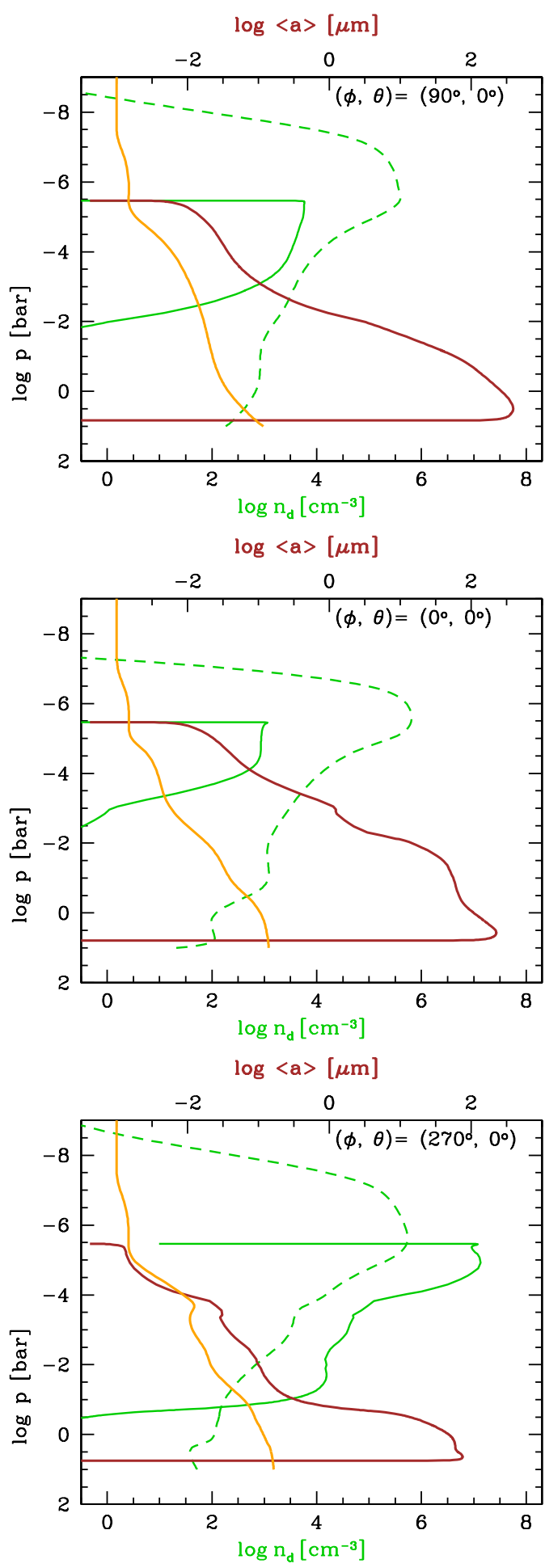

Fig. 14. Characteristic properties of the two cloud particle ensembles: particle number density $\left(\mathrm{n}_{\mathrm{d}}\left[\mathrm{cm}^{-3}\right]\right)$ : mineral particles - green solid, hydrocarbon haze - green dashed. Mean particles size $\langle a\rangle[\mu \mathrm{m}]$ (Eq. (A.3)): mineral particles - brown; hydrocarbon hazes - orange.

compared to a purely gaseous atmosphere, as shown for example in Fig. 20 in Helling et al. (2019b) for the ultra-hot Jupiter HAT-P-7b. In addition to mineral cloud particles, hydrocarbonhaze particles may form in higher atmospheric regions driven by the high-energy fraction of the host star's radiation field. Figure 14 shows that they may appear with a relatively high number density in the upper atmosphere. How would these 

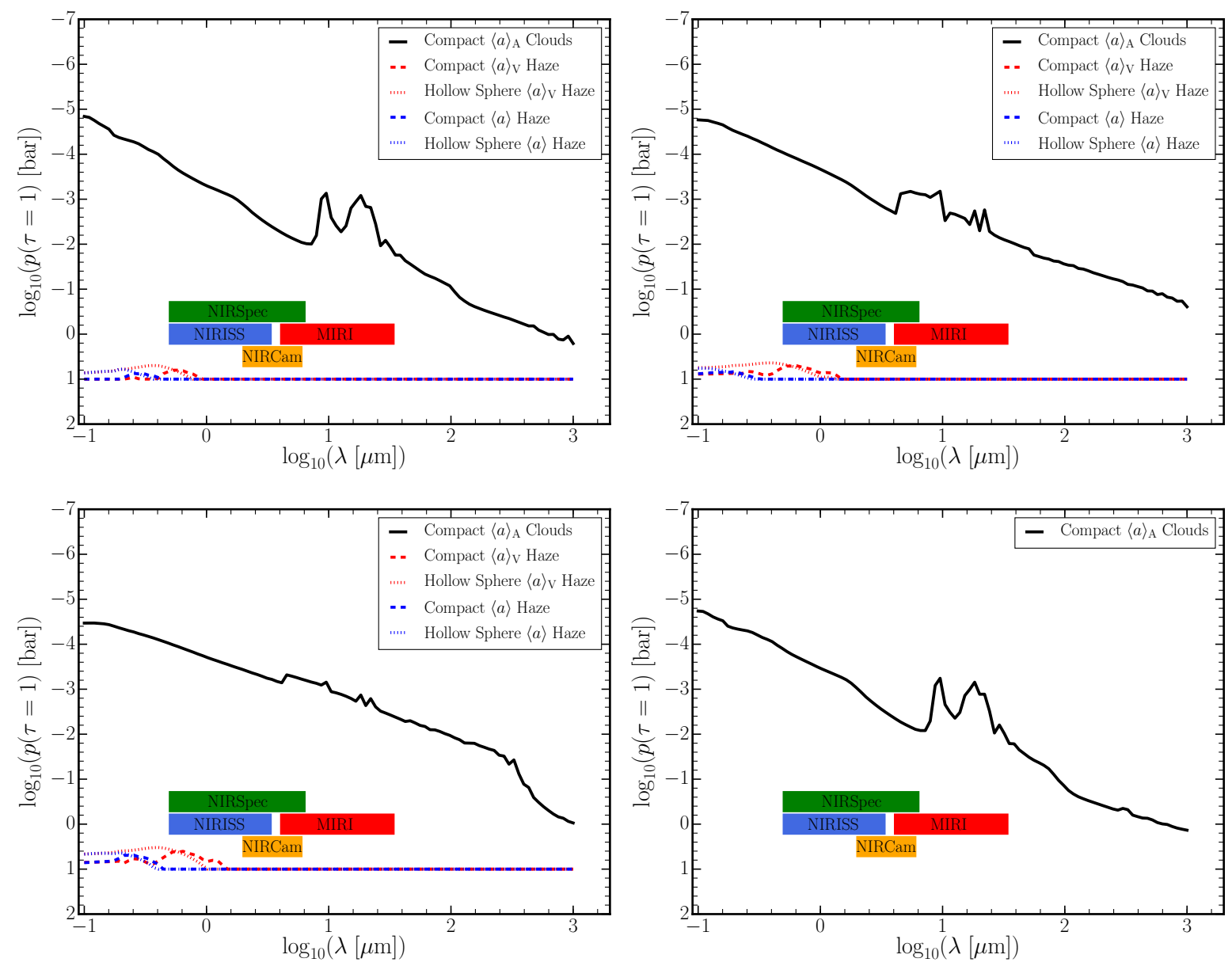

Fig. 15. Atmospheric gas pressure, $p_{\text {gas }}[$ bar], at which the wavelength-dependent vertical optical depth, $\tau(\lambda)$, of the mineral cloud particles (solid black lines) and the hydrocarbon haze particles (red and blue, dashed and dotted lines) reaches unity. Four equatorial profiles are considered: the morning terminator $\left(\phi=270^{\circ}\right.$, top left $)$, the evening terminator $\left(\phi=90^{\circ}\right.$, top right $)$, the substellar point $\left(\phi=0^{\circ}\right.$, bottom left $)$, the anti-stellar point $\left(\phi=180^{\circ}\right.$, bottom right); similar to Figs. 7 and 11. The hydrocarbon hazes are considered to have a tholin opacity (Fig. 16) for compact particles and a distribution of hollow spheres for two averages of particle size. The mineral cloud particles (solid black line) reach $\tau(\lambda)=1$ already at relatively low pressure in the optical but at $p_{\text {gas }} \approx 1$ bar for longer wavelengths, hence, WASP-43b's atmosphere is more transparent in the far-IR. The hydrocarbon hazes remain optically thin. The coloured bars show wavelength ranges covered by the four JWST instruments, based on modes suggested for transmission spectroscopy of exoplanets (Stevenson et al. 2016): MIRI (red) - based on Medium Resolution Spectroscopy modes, NIRCAM (orange) - based on Grism Time Series mode, NIRSPEC (green) - based on Fixed Slit Spectroscopy mode available filters, NIRISS (blue) - based on Single Object Slitless Spectroscopy mode.

two cloud particle populations affect the optical depth of an atmosphere?

To provide a first insight about possible opacity effects of the two cloud particles ensembles, we determine the local gas pressure where the atmosphere reaches an optical depth of 1 along a vertical line of sight from the top of the atmosphere, i.e. $p_{\text {gas }}(\tau(\lambda)=1)$. We apply effective medium and Mie theory for the mixed mineral cloud particles of a mean particle size $\langle a\rangle_{\mathrm{A}}$, number density $n_{\mathrm{d}, \mathrm{A}}=\left(\rho L_{3}\right) /\left(4 \pi\langle a\rangle_{\mathrm{A}}^{3} / 3\right.$ (see Eq. (A.6)), and refractive indices as in Helling et al. (2019b). The hydrocarbon-haze opacities are calculated using the volume averaged haze particle size $\langle a\rangle_{\mathrm{V}}\left(=s_{\mathrm{vol}}\right.$ in Fig. 12) and the respective volume average particle number density $n_{\mathrm{V}}\left(=n_{\mathrm{vol}}\right.$ in Fig. 12), with refractive indices for tholins from Khare et al. (1984) and Ramirez et al. (2002) (Fig. 16). Appendix A demonstrates that uncertainties arise from how the actual mean particle size and the particle number density are calculated, in particular if considering agglomerate particles like in the case of hydrocarbons hazes (see Fig. A.1).

For completeness and for linking to previous calculations, two opacity cases are studied for hydrocarbon hazes: (a) compact haze particles (dashed lines in Fig. 15) and (b) non-compact haze particles by incorporating particle shape effects into the opacity calculations through a distribution of hollow spheres as described in Samra et al. (2020) (dotted lines in Fig. 15). The optical depth is integrated from the top of the respective atmospheres for the hazes $\left(10^{-10}\right.$ [bar]) and clouds $\left(10^{-6}\right.$ [bar] $)$ to the atmospheric gas pressure where the wavelength-dependent optical depth reaches unity. We note that for the haze particles the integrated columns shown here never reach the $\tau=1$. These optical depths are calculated vertically, for a slant geometry the effects can increase (see Fortney 2005).

Figure 15 suggests that hydrocarbon haze particles would appear optically thin in the layers above the mineral cloud particles, i.e. for $p_{\text {gas }}<10^{-4.5}$ bar for $\lambda \approx 0.1 \mu \mathrm{m}$ but up to higher gas pressures of $\approx 1$ bar for the IR wavelength in the terminator profiles. The optical depth of the hydrocarbon hazes is considerably lower than that of the mineral particle clouds such that a wavelength-dependent radius measurement of WASP-43b would be determined by the mineral cloud particles but not by hazes. 


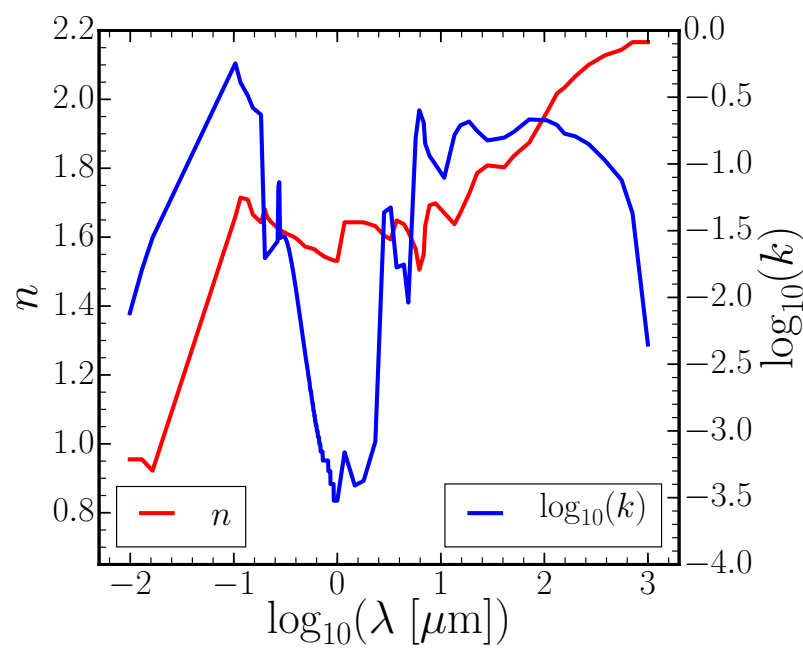

Fig. 16. Refractive index data for Tholins. Data between 0.2 and $1 \mu \mathrm{m}$ is from Ramirez et al. (2002) otherwise from Khare et al. (1984).

If hydrocarbon hazes are optically thin on WASP-43b as suggested here, JWST is likely to observe spectral features of the mineral cloud particles in the IR wavelength of $\approx 15-20 \mu \mathrm{m}$ at the morning terminator $\left(\phi=270^{\circ}\right)$. We would expect more hydrocarbon haze particles if the monomer production rate is higher than what we have assumed in this paper; in the case such that some other species also act as the haze precursors in addition to those considered in this study, $\mathrm{CH}_{4}, \mathrm{HCN}$ and $\mathrm{C}_{2} \mathrm{H}_{2}$.

\section{Conclusion}

- If WASP-43b has an oxygen-rich atmosphere of approximately solar element composition, then WASP-43b can be expected to be covered with mineral clouds on the day- and on the nightside, similar to HD 189733b and HD 209438b.

- Photochemically driven hydrocarbon hazes form on the dayside where the stellar radiation field has the largest effect on the chemistry of the originally oxygen-rich atmospheric gas $(\mathrm{C} / \mathrm{O}<1)$ on WASP-43b. Also, the terminator regions are affected. The effect of UV-driven hydrocarbon haze particles is strongest on the day-side and negligible on the night-side if the horizontal transport is negligible.

- WASP-43b can therefore be expected to have clouds made of two distinct ensembles of particles, mineral particles and hydrocarbon haze particles on the dayside. A gradual change to a mineral-particle only cloud occurs across the terminator regions into the nightside.

- Mineral cloud particles reach size $\approx 100 \mu \mathrm{m}$ in the inner, denser atmospheric regions. Hydrocarbon haze particles may remain smaller, of $\approx 0.1 \mu \mathrm{m}$ in inner, warmer parts of a cloud.

- The cloud and haze particle sizes are subject to precise definition of the term "particles size". The use of different definitions enables to estimate the systematic errors of this cloud property.

- We suggest to search for the spectral features of mineral and of hydrocarbon clusters in the optically thin, low-density regimes of exoplanet atmospheres. We note that similar clusters are studied in AGB star outflows.

- A wavelength-dependent radius measurement of WASP-43b would be determined by the mineral clouds but not by hazes.

- We suggest further to search for mineral absorption features in the $15-20 \mu \mathrm{m}$ spectral range.
Acknowledgements. We thank Peter Woitke for insightful discussions on hydrocarbon hazes. G.V. acknowledges the hospitality of the School of Physics \& Astronomy at the University of St Andrews and the summer student funding from the Royal Astronomical Society. Y.K. and K.L.C. are supported by the European Union's Horizon 2020 Research and Innovation Programme under Grant Agreement 776403. This work has made use of the MUSCLES Treasury Survey High-Level Science Products; doi: 10.17909/T9DG6F. D.S. acknowledges financial support from the Science and Technology Facilities Council (STFC), UK for his PhD studentship (project reference 2093954).

\section{References}

Agúndez, M., Parmentier, V., Venot, O., Hersant, F., \& Selsis, F. 2014, A\&A, 564, A73

Amundsen, D. S., Mayne, N. J., Baraffe, I., et al. 2016, A\&A, 595, A36

Asplund, M., Grevesse, N., Sauval, A. J., \& Scott, P. 2009, ARA\&A, 47, 481

Blecic, J., Harrington, J., Madhusudhan, N., et al. 2014, ApJ, 781, 116

Carone, L., Baeyens, R., Mollière, P., et al. 2020, MNRAS, 496, 3582

Chubb, K. L., Min, M., Kawashima, Y., Helling, Ch., \& Waldmann, I. 2020, A\&A, 639, A3

Decin, L., Richards, A. M. S., Waters, L. B. F. M., et al. 2017, A\&A, 608, A55

Drummond, B., Mayne, N. J., Manners, J., et al. 2018, ApJ, 869, 28

Feng, Y. K., Line, M. R., Fortney, J. J., et al. 2016, ApJ, 829, 52

Fortney, J. J. 2005, MNRAS, 364, 649

France, K., Parke Loyd, R. O., Youngblood, A., et al. 2016, ApJ, 820, 89

Gillon, M., Triaud, A. H. M. J., Fortney, J. J., et al. 2012, A\&A, 542, A4

Hellier, C., Anderson, D. R., Collier Cameron, A., et al. 2011, A\&A, 535, L7

Helling, Ch. 2019, Annu. Rev. Earth Planet. Sci., 47, 583

Helling, Ch., \& Woitke, P. 2006, A\&A, 455, 325

Helling, Ch., Woitke, P., \& Thi, W.-F. 2008, A\&A, 485, 547

Helling, Ch., Woitke, P., Rimmer, P. B., et al. 2014, Life, 4, 142

Helling, Ch., Lee, G., Dobbs-Dixon, I., et al. 2016, MNRAS, 460, 855

Helling, Ch., Gourbin, P., Woitke, P., \& Parmentier, V. 2019a, A\&A, 626, A133

Helling, Ch., Iro, N., Corrales, L., et al. 2019b, A\&A, 631, A79

Hodosán, G., Rimmer, P. B., \& Helling, C. 2016, MNRAS, 461, 1222

Hu, R., Seager, S., \& Bains, W. 2012, ApJ, 761, 166

Irwin, P. G. J., Parmentier, V., Taylor, J., et al. 2020, MNRAS, 493, 106

Kataria, T., Showman, A. P., Fortney, J. J., et al. 2015, ApJ, 801, 86

Kawashima, Y., \& Ikoma, M. 2018, ApJ, 853, 7

Kawashima, Y., \& Ikoma, M. 2019, ApJ, 877, 109

Kawashima, Y., Hu, R., \& Ikoma, M. 2019, ApJ, 876, L5

Khare, B. N., Sagan, C., Arakawa, E. T., et al. 1984, Icarus, 60, 127

Komacek, T. D., Showman, A. P., \& Tan, X. 2017, ApJ, 835, 198

Kreidberg, L., Bean, J. L., Désert, J.-M., et al. 2014, ApJ, 793, L27

Lavvas, P., \& Koskinen, T. 2017, ApJ, 847, 32

Lee, G., Helling, Ch., Dobbs-Dixon, I., \& Juncher, D. 2015, A\&A, 580, A12

Lee, G., Dobbs-Dixon, I., Helling, Ch., Bognar, K., \& Woitke, P. 2016, A\&A, 594, A48

Loyd, R. O. P., France, K., Youngblood, A., et al. 2016, ApJ, 824, 102

Mendonça, J. M., Grimm, S. L., Grosheintz, L., \& Heng, K. 2016, ApJ, 829, 115

Mendonça, J. M., Tsai, S.-m., Malik, M., Grimm, S. L., \& Heng, K. 2018a, ApJ, 869, 107

Mendonça, J. M., Malik, M., Demory, B.-O., \& Heng, K. 2018b, AJ, 155, 150

Molaverdikhani, K., Helling, Ch., Lew, B. W. P., et al. 2020, A\&A, 635, A31

Morello, G., Danielski, C., Dickens, D., Tremblin, P., \& Lagage, P. O. 2019, AJ, 157,205

Parmentier, V., Line, M. R., Bean, J. L., et al. 2018, A\&A, 617, A110

Ramirez, S. I., Coll, P., da Silva, A., et al. 2002, Icarus, 156, 515

Rimmer, P. B., \& Helling, Ch. 2016, ApJS, 224, 9

Rodríguez-Barrera, M. I., Helling, Ch., Stark, C. R., \& Rice, A. M. 2015, MNRAS, 454, 3977

Rodríguez-Barrera, M. I., Helling, Ch., \& Wood, K. 2018, A\&A, 618, A107

Samra, D., Helling, Ch., \& Min, M. 2020, A\&A, 639, A107

Showman, A. P., Fortney, J. J., Lian, Y., et al. 2009, ApJ, 699, 564

Steinrueck, M. E., Parmentier, V., Showman, A. P., Lothringer, J. D., \& Lupu,

R. E. 2019, ApJ, 880, 14

Stevenson, K. B., Désert, J.-M., Line, M. R., et al. 2014, Science, 346, 838

Stevenson, K. B., Lewis, N. K., Bean, J. L., et al. 2016, PASP, 128, 094401

Stevenson, K. B., Line, M. R., Bean, J. L., et al. 2017, AJ, 153, 68

Venot, O., Parmentier, V., Blecic, J., et al. 2020, ApJ, 890, 176

Wang, H., \& Wordsworth, R. 2020, ApJ, 891, 7

Witte, S., Helling, Ch., \& Hauschildt, P. H. 2009, A\&A, 506, 1367

Woitke, P., \& Helling, Ch. 2003, A\&A, 399, 297

Woitke, P., \& Helling, Ch. 2004, A\&A, 414, 335

Youngblood, A., France, K., Parke Loyd, R. O., et al. 2016, ApJ, 824, 101 


\section{Appendix A: Different ways of calculating mean particle sizes}

Defining particle sizes and number densities is easy as long as the particle considered is spherically symmetric. Defining an average particle size becomes challenging if the particles appear with a non-analytic size distribution, such as in clouds. The next step up in complexity for defining particles sizes is if agglomerates form that are made of many monomers, for example, as result of coagulation processes. Such agglomerates could have all sorts of geometries. The definitions of the different representations of the mean particles size $\langle a\rangle[\mathrm{cm}]$ are therefore given here. Equations (A.3) and (A.6) follow from Eqs. (A.2) and (A.4), respectively, by applying $f(a) \mathrm{d} a=f(V) \mathrm{d} V$ and the definition of the dust moments $L_{\mathrm{j}}\left[\mathrm{cm}^{-3} \mathrm{~g}^{-1}\right]\left(\rho L_{\mathrm{j}}\left[\mathrm{cm}^{\mathrm{j}-3}\right]\right)$

$\rho L_{\mathrm{j}}=\int V^{j / 3} f(V) \mathrm{d} V$,

where $V=4 \pi a^{3} / 3$ is the volume of the cloud particle of size $a$.

The weighted averaged particle size $\langle a\rangle$ [cm] (solid brown and orange lines in Figs. 14 and A.1) for a particle size distribution $f(a)\left[\mathrm{cm}^{-3} \mathrm{~cm}^{-1}\right]$

$$
\begin{aligned}
\langle a\rangle & =\frac{\int a f(a) \mathrm{d} a}{\int f(a) \mathrm{d} a} \\
& =\sqrt[3]{\frac{3}{4 \pi}} \frac{L_{1}}{L_{0}} .
\end{aligned}
$$

One can also utilise the moments $L_{3}$ and $L_{2}$ based on the definition in Eq. (A.1) to define a surface averaged mean particle size $\langle a\rangle_{\mathrm{A}}$ (brown and orange dashed lines in Fig. A.1) with $A(a)=4 \pi a^{2}$ :

$$
\begin{aligned}
\langle a\rangle_{\mathrm{A}} & =3 \frac{\int V(a) f(a) \mathrm{d} a}{\int A(a) f(a) \mathrm{d} a} \\
& =\frac{\int a^{3} f(a) \mathrm{d} a}{\int a^{2} f(a) \mathrm{d} a} \\
& =\sqrt[3]{\frac{3}{4 \pi}} \frac{L_{3}}{L_{2}} .
\end{aligned}
$$

We note that $s_{\text {surf }}$ presented in Sect. 6.2 is exactly the same as $\langle a\rangle_{\mathrm{A}}$, which can be recognized by equating $n\left(s_{\mathrm{i}}\right)=f(a) \mathrm{d} a$ in Eq. (64) of Kawashima \& Ikoma (2018) with $n\left(s_{\mathrm{i}}\right)$ being the number density of the cloud particles of size $s_{\mathrm{i}}$. Equation (A.1) results in $\rho L_{2}=(4 \pi / 3)^{2 / 3}\langle a\rangle^{2} n_{\mathrm{d}}, \rho L_{3}=(4 \pi / 3)\langle a\rangle^{3} n_{\mathrm{d}}$ etc. such that the total number of cloud particles is $\int f(V) \mathrm{d} V=n_{\mathrm{d}}$ for a delta-peak-like $f(V)$. From $\rho L_{3}=(4 \pi / 3)\langle a\rangle_{\mathrm{A}}^{3} n_{\mathrm{d}}$ follows then with $\langle a\rangle=\langle a\rangle_{\mathrm{A}}$ that $n_{\mathrm{d}, \mathrm{A}}=\left(\rho L_{3}\right) /\left(4 \pi\langle a\rangle_{\mathrm{A}}^{3} / 3\right)$.

We use another expression for the mean particle size (brown and orange dotted lines in Fig. A.1) as

$$
\begin{aligned}
\sqrt[3]{\left\langle a^{3}\right\rangle} & =\sqrt[3]{\frac{\int a^{3} f(a) \mathrm{d} a}{\int f(a) \mathrm{d} a}} \\
& =\sqrt[3]{\frac{3}{4 \pi} \frac{L_{3}}{L_{0}}}
\end{aligned}
$$

In Fig. A.1, we demonstrate the results for these different definitions of particles sizes (brown and orange lines) as
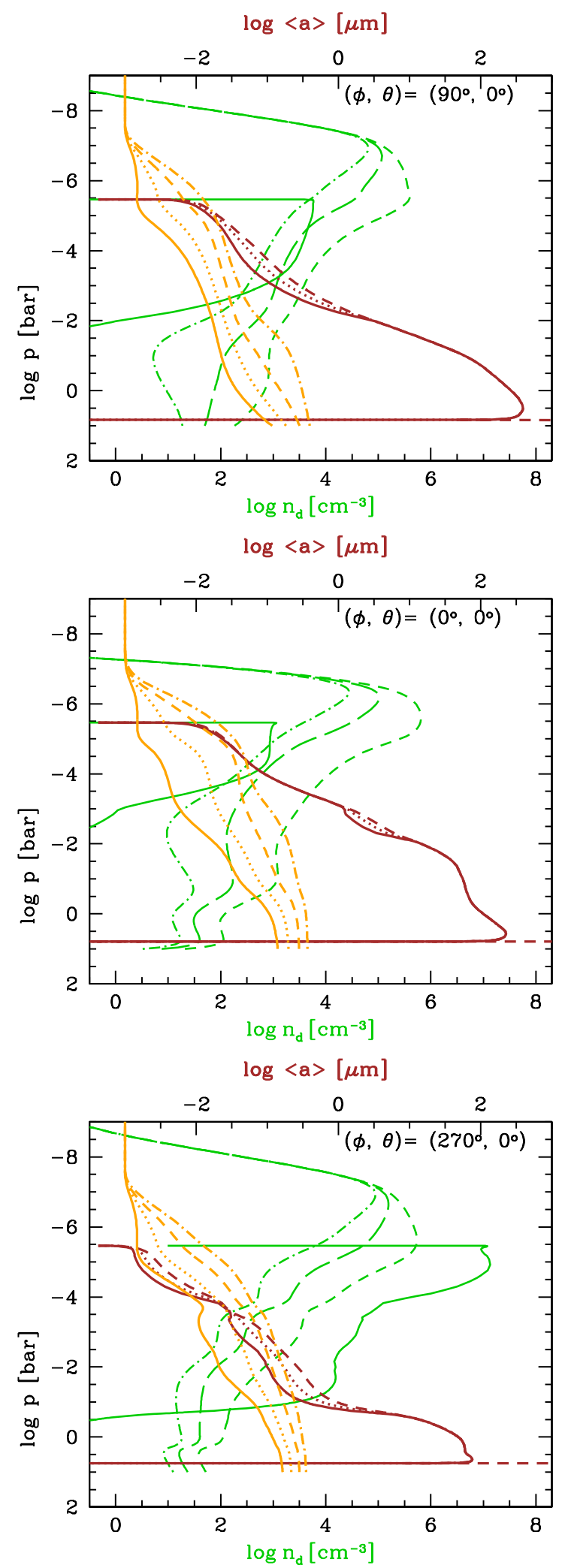

Fig. A.1. Particle number density $\left(\mathrm{n}_{\mathrm{d}}\left[\mathrm{cm}^{-3}\right]\right.$, bottom axis): green solid mineral particles, green dashed - hydrocarbon haze (short dashed - total number density $\sum n\left(a_{i}\right)$ with $a_{i}$ the size of the individual haze particle; long dashed - surface averaged $n_{\text {surf }}$ as in Eq. (65) in, dash-dot volume averaged $n_{\mathrm{Vol}}$ surface as in Eq. (66), both in Kawashima \& Ikoma 2018). Mean particles size $(\langle a\rangle[\mu \mathrm{m}]$, top axis): mineral particles - brown; hydrocarbon hazes - orange $(\langle a\rangle$ (Eq. (A.3)) - solid lines; $\langle a\rangle_{\mathrm{A}}=s_{\text {surf }}$ (Eq. (A.6)) - dashed lines; $\sqrt[3]{\left\langle a^{3}\right\rangle}$ (Eq. (A.8)) - dotted lines, $\langle a\rangle_{\mathrm{V}}=s_{\mathrm{vol}}$ as in Eq. (38) in Kawashima \& Ikoma 2018 - dash-dot). 
result of two different modelling methods. The hydrocarbon haze model of Kawashima \& Ikoma (2018) which was applied in this paper uses a binning method in order to follow the growth of the agglomerates. The method of Woitke \& Helling (2003, 2004), Helling \& Woitke (2006), Helling et al. (2008) that we used to calculate the mineral cloud particles solves differential moment method where the moments are weighted integrals over the particle size distribution as defined in Eq. (A.1). Applying the same definitions (Eqs. (A.2)-(A.8)) to the results from both methods allows for a more scientific comparison between the resulting values of the mean particles sizes (or any other property). It enables us further to provide information about what uncertainties cloud particle sizes should be expected. Comparing the mean particles sizes derived by different formula further demonstrate that the definition of the particles size becomes increasingly more challenging if particleparticle processes are modelled as in coagulation simulations. This is also the case when defining particle number densities (green dashed lines in Fig. A.1): the volume averaged particles number density, which is of relevance for opacity calculations, can differ by two orders of magnitudes from the number density that sums up all existing particles as $\sum_{i} n\left(a_{i}\right)$ with $a_{i}\left(V_{\mathrm{i}}\right)$ the size of the individual haze particle of volume $V_{\mathrm{i}}$.

\section{Appendix B: Supplementary plots}

Here we provide the detailed results that have been used to produce the more condensed results of the previous sections.

Input for photochemical haze calculation for WASP-43b. Figure B. 1 provides the $1 \mathrm{D}\left(T_{\mathrm{gas}}, p_{\text {gas }}, K_{\mathrm{zz}}\right)$-profiles that have been used for the photochemical gas and haze calculations in Sect. 6.

Details for mineral cloud particle composition. Figure B.2 details how the material composition of the mineral cloud particles changes vertically for the four example $1 \mathrm{D}\left(T_{\mathrm{gas}}, p_{\mathrm{gas}}\right)$ profiles. The details in Fig. B. 2 are the base for the 5 material categories in Fig. 7 grouped as follows:

- silicates $\left(\mathrm{MgSiO}_{3}[\mathrm{~s}], \quad \mathrm{Mg}_{2} \mathrm{SiO}_{4}[\mathrm{~s}], \quad \mathrm{Fe}_{2} \mathrm{SiO}_{4}[\mathrm{~s}]\right.$

$\left.\mathrm{CaSiO}_{3}[\mathrm{~s}]\right)$;

- metal oxides $\left(\mathrm{MgO}[\mathrm{s}], \mathrm{SiO}[\mathrm{s}], \mathrm{SiO}_{2}[\mathrm{~s}], \mathrm{FeO}[\mathrm{s}], \mathrm{Fe}_{2} \mathrm{O}_{3}[\mathrm{~s}]\right.$; $\left.\mathrm{CaTiO}_{3}[\mathrm{~s}]\right)$;

- high temperature condensates $\left(\mathrm{TiO}_{2}[\mathrm{~s}], \mathrm{Fe}[\mathrm{s}], \mathrm{Al}_{2} \mathrm{O}_{3}[\mathrm{~s}]\right)$;

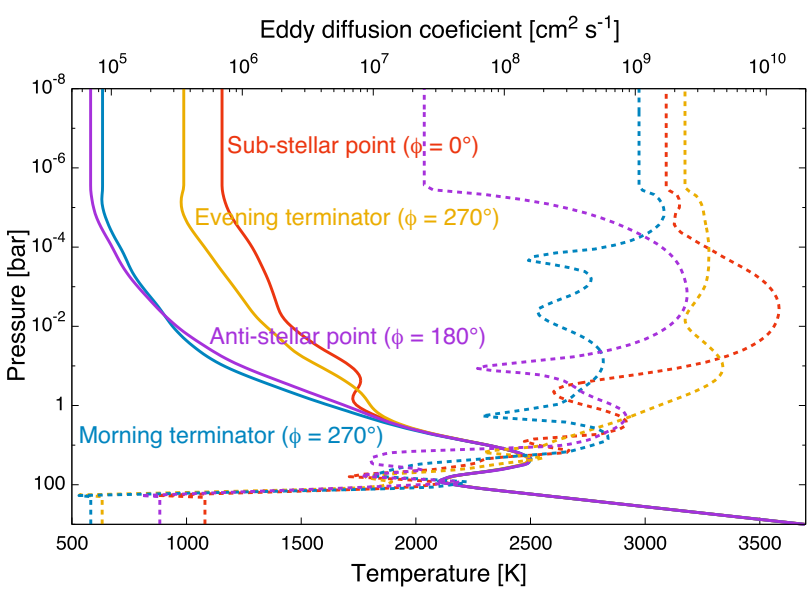

Fig. B.1. 1D profiles ( $T_{\mathrm{gas}}-$ solid lines, $K_{\mathrm{zz}}-$ dotted lines) used to study the formation of hydrocarbon hazes at three distinct locations in the atmosphere of WASP-43b. These profiles are isothermaly extended into higher altitudes than those used in Fig. 2.

- carbon $(\mathrm{C}[\mathrm{s}])$; and

- salts (here $\mathrm{KCl}[\mathrm{s}]$ ).

Details of chemical equilibrium gas-phase composition for WASP-43b. Figure B.3 summarised the 15 most abundant molecules for the WASP-43b substellar and anti-stellar point and the two equatorial terminators. The most abundant molecules in chemical equilibrium are $\mathrm{CO}$ and $\mathrm{H}_{2} \mathrm{O}$, after $\mathrm{H}_{2}$ and $\mathrm{He} . \mathrm{H}_{2}, \mathrm{CO}$ and $\mathrm{H}_{2} \mathrm{O}$ will be affected by photochemical reactions as demonstrated in Fig. 11. The next abundant molecule is $\mathrm{SiO}$ on the dayside but $\mathrm{CH}_{4}$ on the nightside in chemical equilibrium. Both are not affected by photochemistry, $\mathrm{SiO}$ because it is not considered in the photochemical code and $\mathrm{CH}_{4}$ because no stellar photons reach the nightside in our present calculations. Molecules like $\mathrm{AlOH}, \mathrm{CO}_{2}$ and $\mathrm{TiO}$ are considerably less abundant. The abundance of $\mathrm{AlOH}$ and $\mathrm{TiO}$ is further affected by element depletion through mineral cloud formation.

Figure B.4 gives an account of the atomic and molecular ion species for the WASP-43b substellar and anti-stellar point and the two equatorial terminators. The most abundant ions are $\mathrm{K}^{+}$, $\mathrm{Na}^{+}$and $\mathrm{Ca}^{+}$followed by $\mathrm{H}^{-}$. Photochemistry does not affect $\mathrm{H}^{-}$ in our simulations. 
Ch. Helling et al.: Mineral cloud and hydrocarbon haze particles in the atmosphere of the hot Jupiter JWST target WASP-43b
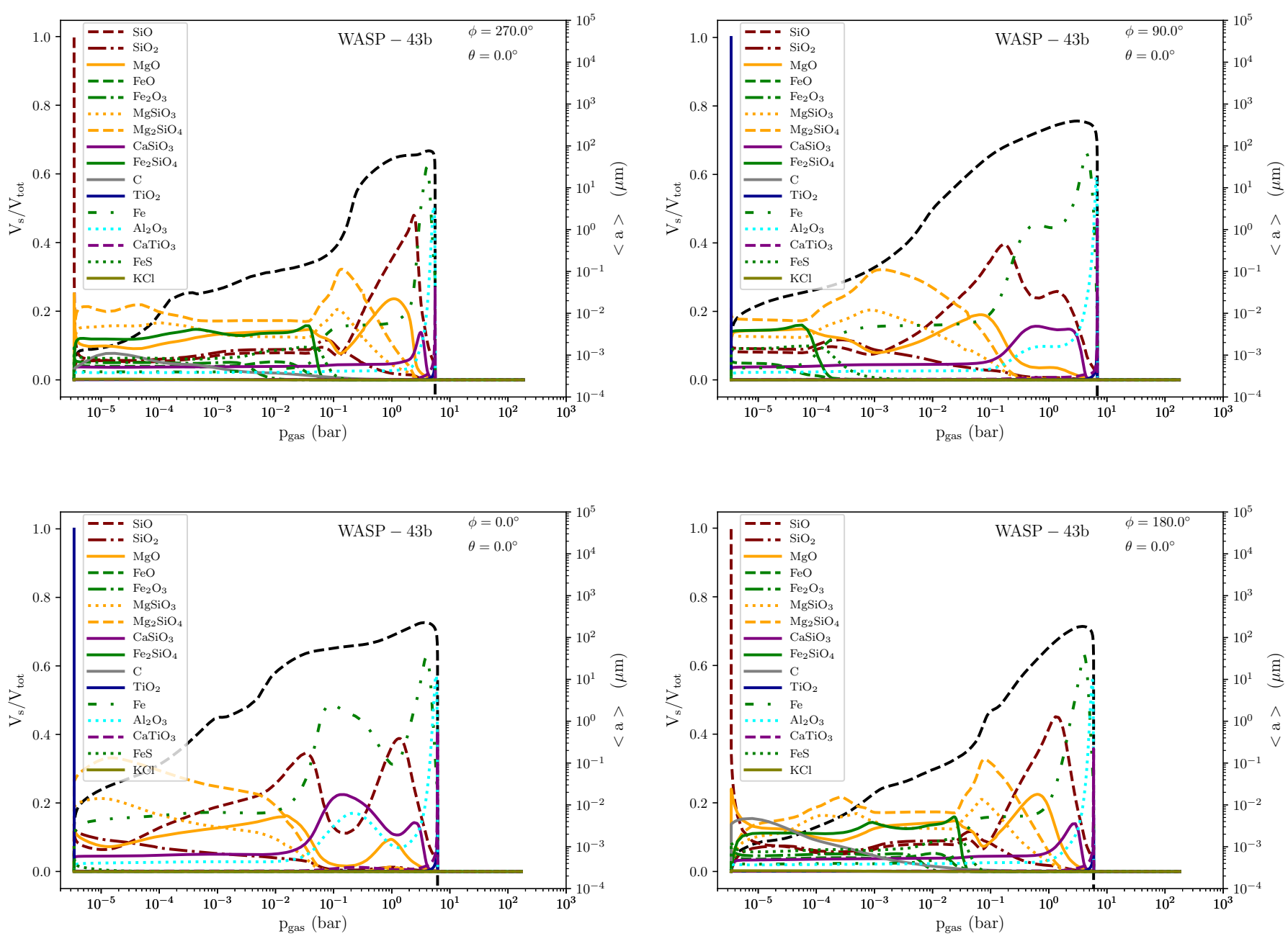

Fig. B.2. Detailed material volume fraction for the morning terminator (top left), evening terminator (top right), sub-stellar point (bottom left), anti-stellar point (bottom right). 

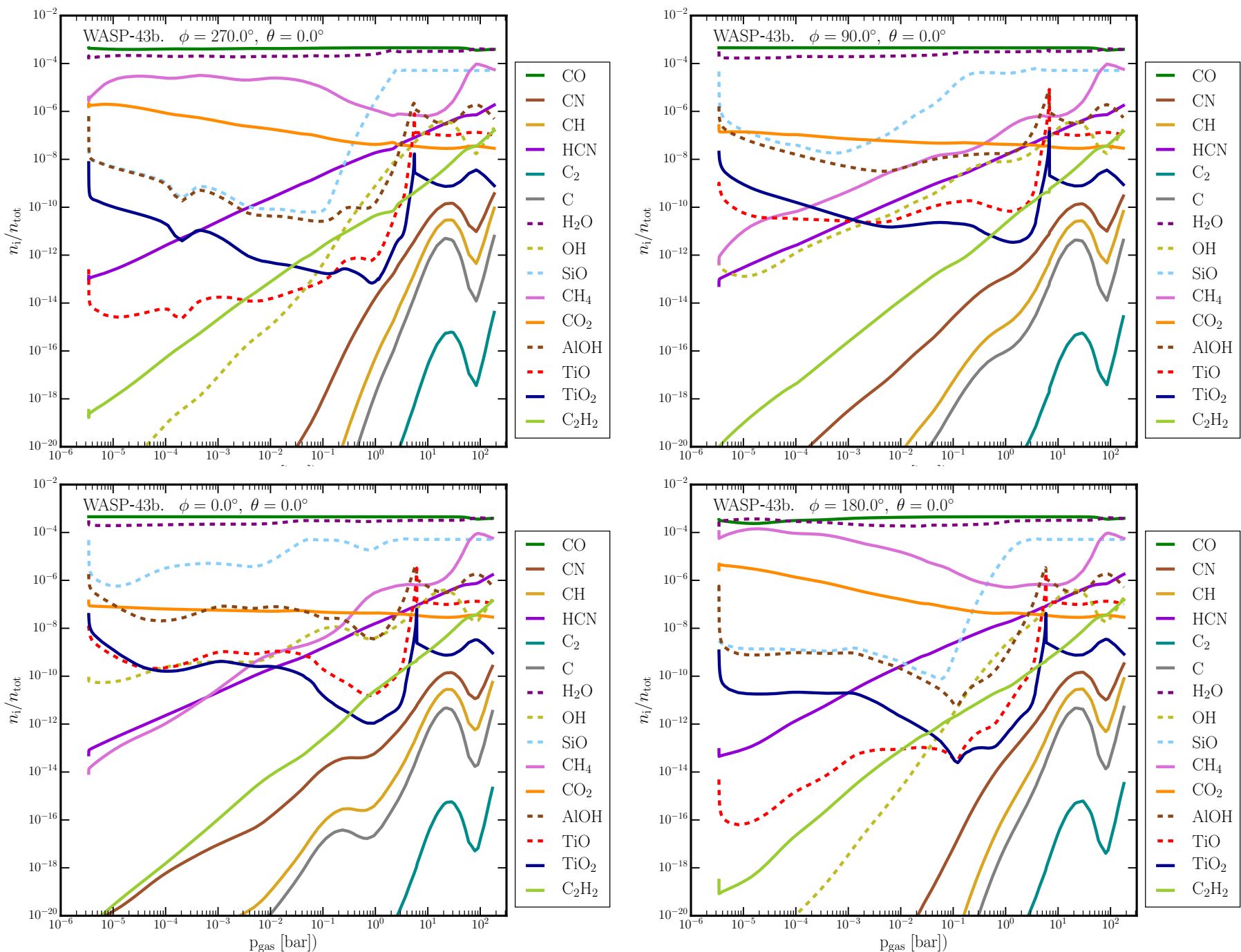

Fig. B.3. Concentrations, $n_{\mathrm{i}} / n_{\text {tot }}$, for the 15 most abundant molecules (after $\mathrm{H}_{2 /} \mathrm{H}$ ), in chemical equilibrium based on the cloud-depleted element abundances on WASP-43b. Top left: morning terminator, top right: evening terminator, bottom left: sub-stellar point, bottom right: anti-stellar point. 
Ch. Helling et al.: Mineral cloud and hydrocarbon haze particles in the atmosphere of the hot Jupiter JWST target WASP-43b
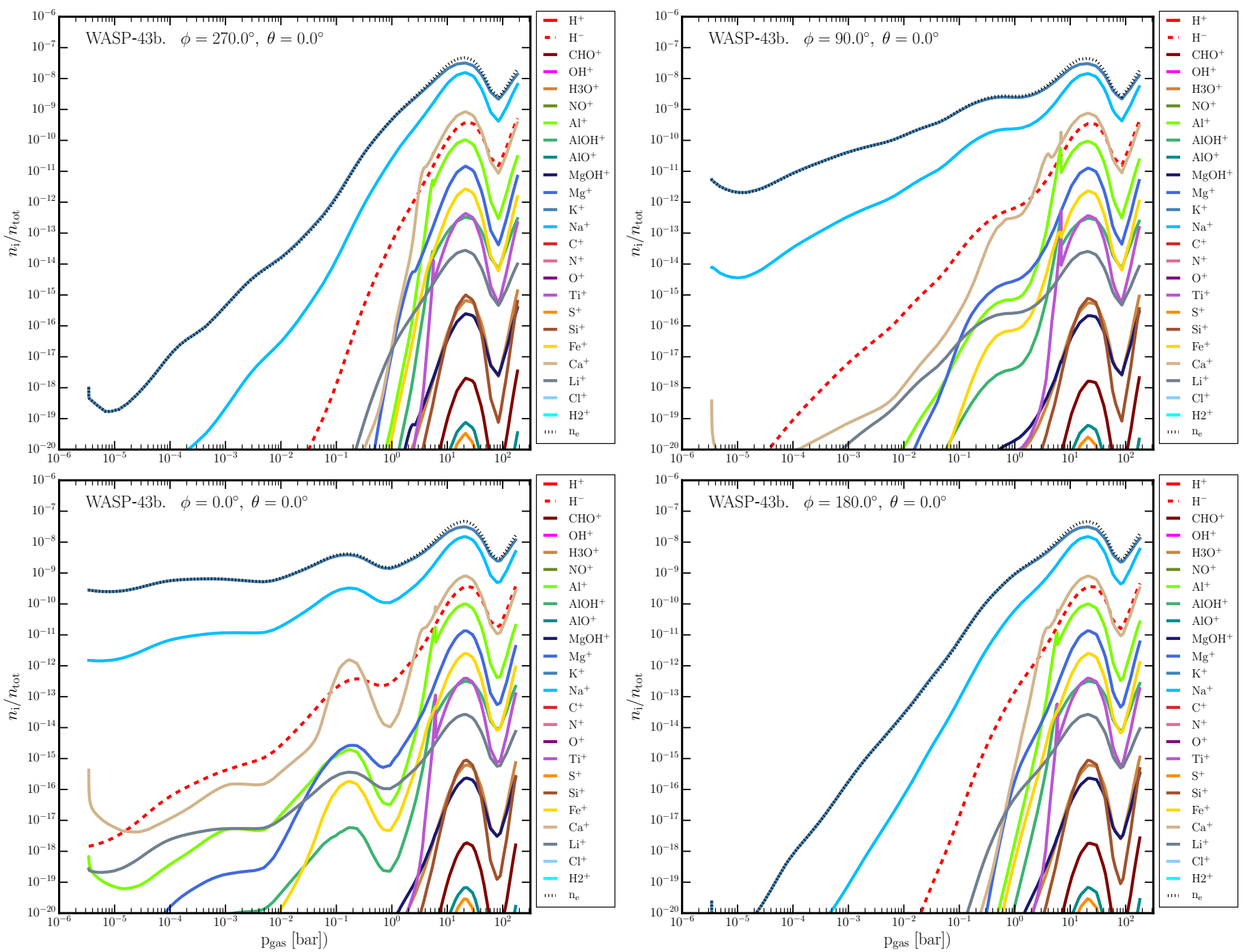

Fig. B.4. Ion concentrations, $n_{\mathrm{i}} / n_{\mathrm{tot}}$, in thermal equilibrium for the four selected 1D profiles based on the cloud-depleted element abundances. Top left: morning terminator, top right: evening terminator, bottom left: sub-stellar point, bottom right: anti-stellar point. The electron number density is shown as black dash line. 